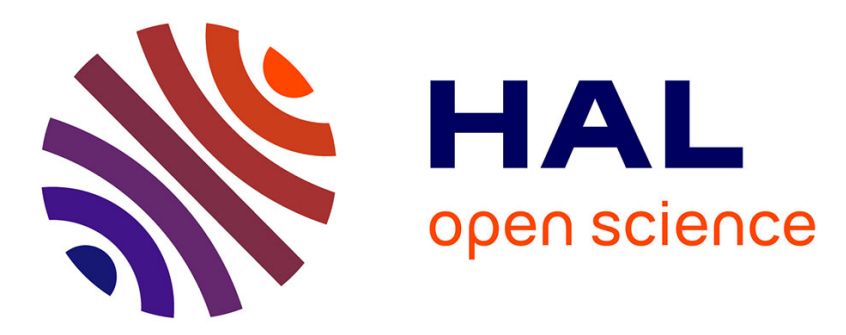

\title{
Homogenization of a space frame as a thick plate: Application of the Bending-Gradient theory to a beam lattice
}

\author{
Arthur Lebée, Karam Sab
}

\section{- To cite this version:}

Arthur Lebée, Karam Sab. Homogenization of a space frame as a thick plate: Application of the Bending-Gradient theory to a beam lattice. Computers \& Structures, 2013, pp.88-101. hal-00938967

\section{HAL Id: hal-00938967 \\ https://hal-enpc.archives-ouvertes.fr/hal-00938967}

Submitted on 29 Jan 2014

HAL is a multi-disciplinary open access archive for the deposit and dissemination of scientific research documents, whether they are published or not. The documents may come from teaching and research institutions in France or abroad, or from public or private research centers.
L'archive ouverte pluridisciplinaire HAL, est destinée au dépôt et à la diffusion de documents scientifiques de niveau recherche, publiés ou non, émanant des établissements d'enseignement et de recherche français ou étrangers, des laboratoires publics ou privés. 


\title{
Homogenization of a space frame as a thick plate: application of the Bending-Gradient theory to a beam lattice
}

\author{
A. Lebée*, K. Sab \\ Université Paris-Est, Laboratoire Navier (UMR 8205), CNRS, ENPC, IFSTTAR \\ F-77455 Marne-la-Vallée
}

\begin{abstract}
The Bending-Gradient theory for thick plates is the extension to heterogeneous plates of Reissner-Mindlin theory originally designed for homogeneous plates. In this paper the Bending-Gradient theory is extended to in-plane periodic structures made of connected beams (space frames) which can be considered macroscopically as a plate. Its application to a square beam lattice reveals that classical Reissner-Mindlin theory cannot properly model such microstructures. Comparisons with exact solutions show that only the BendingGradient theory captures second order effects in both deflection and local stress fields.
\end{abstract}

Keywords: Plate theory, Higher-order Models, Homogenization, Periodic plates, Space frame

\section{Introduction}

The classical theory of plates, known also as Kirchhoff-Love plate theory is based on the assumption that the normal to the mid-plane of the plate remains normal after transformation. This theory is also the first order of the asymptotic expansion with respect to the thickness [1]. Thus, it presents a good theoretical justification and was soundly extended to the case of periodic plates [2]. It enables to have a first order estimate of the macroscopic deflection as well as local stress fields. In most applications the first order deflection is accurate enough. However, this theory does not capture the local effect of shear forces on the microstructure because shear forces are one higher-order derivative of the bending moment in equilibrium equations $\left(Q_{\alpha}=M_{\alpha \beta, \beta}\right)$.

Because shear forces are part of the macroscopic equilibrium of the plate, their effect is also of great interest for engineers when designing structures. However, modeling properly the action of shear forces is still a controversial issue. Reissner [3] suggested a model for homogeneous plates based on a parabolic distribution of transverse shear stress through the thickness (Reissner-Mindlin theory). This model performs well for homogeneous plates and gives more natural boundary conditions than those of Kirchhoff-Love theory. Thus, it is appreciated by engineers and broadly used in applied mechanics. However, the direct extension of this model to more complex microstructures raised many difficulties. Many suggestions were made for laminated plates [4, 5, 6, 7, 8, 9] as well as in-plane periodic plates [10, 11], leading to more complex models.

Revisiting the approach from Reissner [3] directly with laminated plates, Lebée and Sab [12, 13] showed that the transverse shear static variables which come out when the plate is heterogeneous are not shear forces $Q_{\alpha}$ but the full gradient of the bending moment $R_{\alpha \beta \gamma}=M_{\alpha \beta, \gamma}$. Using conventional variational tools, they derived a new plate theory - called Bending-Gradient theory - which is actually turned into Reissner-Mindlin theory when the plate is homogeneous. This new plate theory is seen by the authors as an extension of Reissner's theory to heterogeneous plates which preserves most of its simplicity. Originally designed for laminated plates, it was also extended to in-plane periodic plates using averaging considerations such as Hill-Mandel principle and successfully applied to sandwich panels [14, 15].

In order to give a more comprehensive illustration of the features of this new theory, we extend its homogenization scheme to space frames (Section 21). Space frames are large roofings made of many identical unit-cells. Numerous illustration are given in Buckminster Fuller's achievements. In this work, a space 
frame is a unit-cell made of connected beams periodically reproduced in a plane and which "from far" can be considered as a plate. Many devices fall into this category: space trusses [16, 17, 18, 19], tensegrity, nexorade [20], gridshells [21], lattices, expanded metal, gratings, etc.

Let's point out that very few methods exist in the literature for deriving a thick plate macroscopic model when the microstructure is made of structural elements. Clearly, theories for laminates are not suitable for the present microstructure and we need an approach which takes into account the periodicity of the plate. There is also a large literature dedicated to the homogenization of beam lattices (also referred as discrete medias [22, 23]). However, these approaches lead only to 3D or in-plane macroscopic models. Finally, the work from Lewiski [10] and Cecchi and Sab [1] suggest homogenization techniques for a thick plate which apply only to microstructures with the classical Cauchy's continuum. Hence, in this respect the present approach is innovative.

One can argue that from an engineering point of view, the full simulation of the lattice remains affordable in terms of computation and will directly lead to more accurate results. However, because these structures are periodic, their design is often based on the assessment of a single unit-cell. Engineers check if this unit-cell sustains highest loads in critical areas and then, set the design for the entire roof. Having a single unit-cell reproduced many times enables returns to scale and reduces costs. Such an approach requires a good knowledge of local stress fields in the unit-cell generated by macroscopic loadings, independently of the configuration of the plate, which is exactly the purpose of homogenization techniques. Hence these approaches are complementary of full simulations.

In Section 3 we consider a square beam lattice (Figure 1) and apply both Bending-Gradient and Cecchi and Sab 11] Reissner-Mindlin homogenization scheme. This very simple pattern will enable the derivation of closed-form solutions of the auxiliary problems which are easy to interpret even if the approach can handle 3D geometries. Let us already point out that, because of the patterns symmetries, the Reissner-Mindlin shear forces stiffness $\underset{\sim}{\boldsymbol{F}}\left(Q_{\alpha}=F_{\alpha \beta} \gamma_{\beta}\right.$, where $\gamma_{\alpha}$ is Reissner-Mindlin shear strain) is necessarily isotropid 1 : $F_{\alpha \beta}=F \delta_{\alpha \beta}$. However, the pattern must be somehow sensitive to the bending orientation.

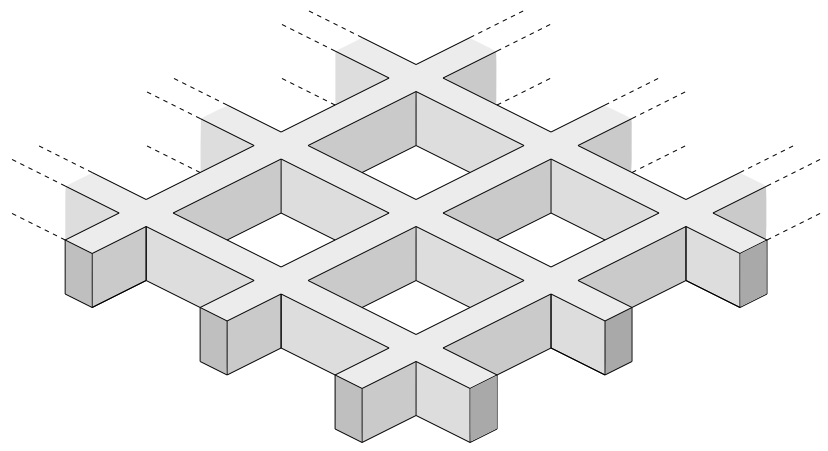

Figure 1: A square beam lattice

In order to check this prediction, a comparison with exact solutions of the cylindrical bending of the lattice in two orientations is performed in Section 4. It reveals that only the Bending-Gradient theory is able to capture second order effects both in terms of deflection and local stress fields.

\section{Homogenization of a periodic space frame as a thick plate}

Full details about the Bending-Gradient plate theory are provided in [12, 13, 14]. In this section we recall its main features and derive a homogenization scheme dedicated to space frames.

\footnotetext{
${ }^{1}$ Two orthogonal direction of orthotropy and invariance under $90^{\circ}$ in-plane rotation
} 


\subsection{Summary of the Bending-Gradient plate model}

We consider a linear elastic plate which mid-plane is the $2 \mathrm{D}$ domain $\omega \subset \mathbb{R}^{2}$. Cartesian coordinates $\left(x_{1}, x_{2}, x_{3}\right)$ in the reference frame $\left(\underline{\boldsymbol{e}}_{1}, \underline{\boldsymbol{e}}_{2}, \underline{\boldsymbol{e}}_{3}\right)$ are used to describe macroscopic fields. At this stage, the microstructure of the plate is not specified.

The membrane stress $N_{\alpha \beta}$, the bending moment $M_{\alpha \beta}$, and shear forces $Q_{\alpha}(\alpha, \beta, \gamma \ldots=1,2)$ are the usual generalized stresses for plates. Moreover, the main feature of the Bending-Gradient theory is the introduction of an additional static unknown: the gradient of the bending moment $R_{\alpha \beta \gamma}=M_{\alpha \beta, \gamma}$. The 2D third-orde1 2 tensor $\boldsymbol{R}$ complies with the following symmetry: $R_{\alpha \beta \gamma}=R_{\beta \alpha \gamma}$. It is possible to derive shear forces $\boldsymbol{Q}$ from $\boldsymbol{R}$ with: $Q_{\alpha}=R_{\alpha \beta \beta}$.

The full bending gradient $\underline{\boldsymbol{R}}$ has six components (taking into account symmetries of indices) whereas $\underline{\boldsymbol{Q}}$ has two components. Thus, using the full bending gradient as static unknown introduces four additional static unknowns. More precisely: $R_{111}$ and $R_{222}$ are respectively the cylindrical bending part of shear forces $Q_{1}$ and $Q_{2}, R_{121}$ and $R_{122}$ are respectively the torsion part of these shear forces and $R_{112}$ and $R_{221}$ are linked to strictly self-equilibrated stresses. Equilibrium equations and stress boundary conditions are detailed in Appendix A . They are very similar to those of Reissner-Mindlin theory where $Q_{\alpha}=M_{\alpha \beta, \beta}$ is replaced by $R_{\alpha \beta \gamma}=M_{\alpha \beta, \gamma}$.

The main difference between Reissner-Mindlin and Bending-Gradient plate theories is that the latter enables the distinction between each component of the gradient of the bending moment whereas they are mixed into shear forces with Reissner-Mindlin theory.

Generalized stresses $N_{\alpha \beta}, M_{\alpha \beta}$ and $R_{\alpha \beta \gamma}$ work respectively with the dual strain variables: $e_{\alpha \beta}$, the conventional membrane strain, $\chi_{\alpha \beta}$ the curvature and $\Gamma_{\alpha \beta \gamma}$ the third order tensor related to generalized shear strains. These strain fields must comply with the compatibility conditions and boundary conditions detailed in Appendix A.

Finally, assuming uncoupling between $(\underset{\sim}{N}, \underset{\sim}{M})$ and $\boldsymbol{R}$ (see 12,14$])$, the Bending-Gradient plate constitutive equations are written as:

$$
\left\{\begin{array}{l}
\underset{\sim}{\boldsymbol{N}}=\underset{\sim}{\boldsymbol{A}}: \underset{\sim}{\boldsymbol{e}}+\underset{\sim}{\boldsymbol{B}}: \underset{\sim}{\boldsymbol{\chi}} \\
\underset{\sim}{\boldsymbol{M}}=\underset{\sim}{\boldsymbol{T}}: \underset{\sim}{\boldsymbol{T}}+\underset{\sim}{\boldsymbol{D}}: \underset{\sim}{\boldsymbol{\sim}} \\
\underset{\sim}{\boldsymbol{f}}: \boldsymbol{R}
\end{array}\right.
$$

where $\underset{\sim}{\boldsymbol{A}}, \underset{\approx}{\boldsymbol{B}}, \underset{\sim}{\boldsymbol{D}})$ are conventional Kirchhoff-Love fourth order stiffness tensors and ":" is the double contraction product. The generalized shear compliance tensor $f$ is a sixth order tensol 3 and ":" denotes a triple contraction product: $\boldsymbol{f}: \underline{\boldsymbol{R}}=\left(f_{\alpha \beta \gamma \delta \epsilon \zeta} R_{\zeta \epsilon \delta}\right)$.

In some cases, the Bending-Gradient is turned into a Reissner-Mindlin plate model. This is the case for homogeneous plates. In order to estimate the difference between both plate models we defined the isotropic projection of the Bending-Gradient stress energy density on a Reissner-Mindlin one in [12]. According to this projection, the Reissner-Mindlin part of $\underset{f}{ }$ is:

$$
\underline{\boldsymbol{f}}^{R M}=\left(\frac{2}{3} \underset{\sim}{\boldsymbol{i}} \cdot \underset{\sim}{\boldsymbol{i}}\right): \underset{\sim}{\boldsymbol{f}}:\left(\frac{2}{3} \underset{\sim}{\boldsymbol{i}} \cdot \underset{\sim}{\boldsymbol{i}}\right)
$$

where $\boldsymbol{i}$ is the identity for in-plane elasticity tensors $\left(i_{\alpha \beta \gamma \delta}=\frac{1}{2}\left(\delta_{\alpha \gamma} \delta_{\beta \delta}+\delta_{\alpha \delta} \delta_{\beta \gamma}\right)\right)$ and $\underset{\sim}{\boldsymbol{i}} \cdot \underset{\boldsymbol{i}}{\boldsymbol{i}}=\left(i_{\alpha \beta \gamma \eta} i_{\eta \delta \epsilon \zeta}\right)$ is a sixth order tensor. Consequently, we suggested the following relative distance between the Bending-Gradient

\footnotetext{
${ }^{2}$ Vectors and higher-order tensors are boldfaced and different underlinings are used for each order: vectors are straight underlined, $\boldsymbol{u}$. Second order tensors are underlined with a tilde: $\boldsymbol{M}$. Third order tensors are underlined with a parenthesis: $\boldsymbol{R}$. Fourth order tensors are are doubly underlined with a tilde: $\underset{\sim}{D}$. Sixth order tensors are doubly underlined with a parenthesis: f.

${ }^{3} f_{\alpha \beta \gamma \delta \epsilon \zeta}$ follows major symmetry: $f_{\alpha \beta \gamma \delta \epsilon \zeta}=f_{\zeta \epsilon \delta \gamma \beta \alpha}$ and minor symmetry $f_{\alpha \beta \gamma \delta \epsilon \zeta}=f_{\beta \alpha \gamma \delta \epsilon \zeta}$. Thus there are only 21 independent components.
} 
and the Reissner-Mindlin stress energy densities:

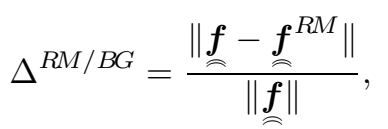

$$
\begin{aligned}
& \text { where }\|\boldsymbol{f}\|=\sqrt{f_{\alpha \beta \gamma \delta \epsilon \zeta} f_{\alpha \beta \gamma \delta \epsilon \zeta}}
\end{aligned}
$$

The scalar $\Delta^{R M / B G}$ gives an estimate of the pure warping fraction of the shear stress energy and is a criterion for assessing the need for the Bending-Gradient model: $0 \leq \Delta^{R M / B G}<1$. When the plate constitutive equation reduces to a Reissner-Mindlin one, we have exactly $\Delta^{R M / B G}=0$ and both theories are strictly identical. Finally, if $\underset{\sim}{\boldsymbol{f}}$ writes as: $\underset{\sim}{\boldsymbol{f}}=\underset{\sim}{\boldsymbol{i}} \cdot \underset{\sim}{\boldsymbol{f}} \cdot \underset{\sim}{\boldsymbol{i}}$ where $\underset{\sim}{\boldsymbol{f}}$ is a second order tensor, then $\Delta^{R M / B G}=0$ and the Reissner-Mindlin compliance is exactly $f$.

The constitutive tensors $\underset{\sim}{\boldsymbol{A}} \underset{\sim}{\boldsymbol{B}}, \underset{\sim}{\boldsymbol{D}}$ and $\boldsymbol{f}$ are function of the plate microstructure. Their derivation is based on the homogenization scheme discussed in the next section.

\subsection{Homogenization scheme}

When the plate is laminated, closed-form expression for $\underset{\sim}{\boldsymbol{A}} \underset{\sim}{\boldsymbol{\sim}}, \underset{\sim}{\boldsymbol{D}}$ and $\boldsymbol{f}$ where derived in [12]. When the plate is periodic those tensors must be derived using an homogenization procedure. This procedure relies on the fundamental assumption that loadings as well as macroscopic variables $(\underset{\sim}{\boldsymbol{M}}, \boldsymbol{M}, \boldsymbol{R})$ varies slowly compared to the size of the microstructure (the unit-cell). This means that at the scale of the unit-cell the macroscopic fields can be considered as uniform. Hence, it is possible to define auxiliary problems which enable the derivation of the actual microscopic fields as a linear superposition of macroscopic fields. Once this is done, it is straightforward to take the average of the elastic energy stored inside the unit-cell and equate it to the macroscopic elastic energy density (namely the macroscopic constitutive equation). This analogy between micro and macroscopic energy is usually called Hill-Mandel principle. When the plate microstructure is constituted of a 3D continuum, the auxiliary problems were introduced in [14]. In the present case, we consider periodic plates which unit-cell is constituted of connected beams. This necessitates the adaptation of the homogenization scheme for 3D continuum. In this section, we first describe the unitcell and the related beam model. Then the Kirchhoff-Love auxiliary problem which enables the derivation of $\underset{\sim}{\boldsymbol{A}}, \underset{\widetilde{\sim}}{\boldsymbol{B}}, \underset{\sim}{\boldsymbol{D}}$ is adjusted to this type of plate. Finally, the Bending-Gradient auxiliary problem is derived through a direct approach and defines the compliance $\boldsymbol{f}$.

\subsubsection{The unit-cell of the space frame}

In this section, we give a general description of the space frame and set the main assumptions. We also introduce some useful definitions.

We consider an in-plane periodic plate which unit-cell is made of an assembly of connected beams Figure 22. The unit-cell reference frame is $\left(\underline{\boldsymbol{e}}_{1}, \underline{\boldsymbol{e}}_{2}, \underline{\boldsymbol{e}}_{3}\right)$ associated with the local coordinate $\boldsymbol{y}=\left(y_{1}, y_{2}, y_{3}\right)$.

There are $n_{b}$ beams constituting the unit-cell intersecting at nodes (see Figure 2). Each beam connects two nodes through a path $\gamma_{k}$. The collection of $\gamma_{k}$ is $\Gamma=\cup_{k=1}^{n_{b}} \gamma_{k}$. Without loss of generality, we assume here a perfect connection at nodes (fully clamped). Furthermore, a subset of these nodes are pairs of periodicity nodes: they connect the unit-cell to its neighbors. Thus interior nodes are denoted $I_{i}\left(i=1, n_{i}\right)$ and periodicity pairs $P_{j}$ and $\bar{P}_{j},\left(j=1, n_{p}\right)$.

Local beam equations. The local reference frame along each beam is denoted $(\underline{\boldsymbol{t}}, \underline{\boldsymbol{n}}, \underline{\boldsymbol{b}})$ associated with the coordinate $\underline{s}=\left(s_{1}, s_{2}, s_{3}\right)$. The unit vector $\underline{\boldsymbol{t}}$ is oriented in the direction of the neutral axis of the beam. For the sake of simplicity, beams are assumed rectilinear and follows St Venant's approximation with uniform torsion. Consequently, the resultant force $\underline{\boldsymbol{r}}$ and the moments $\underline{\boldsymbol{m}}$ are defined as follows:

$$
\underline{\boldsymbol{r}}=\int_{\mathcal{S}} \underline{\boldsymbol{\sigma}}^{B} \cdot \underline{\boldsymbol{t}} d \mathcal{S} \text { and } \underline{\boldsymbol{m}}=\int_{\mathcal{S}} \underline{\boldsymbol{s}} \times\left(\underline{\boldsymbol{\sigma}}^{B} \cdot \underline{\boldsymbol{t}}\right) d \mathcal{S}
$$

where $\mathcal{S}$ is the beam section, $\boldsymbol{\sigma}_{\sim}^{B}$ is the beam's local 3D stress and $\times$ denotes the cross product. 


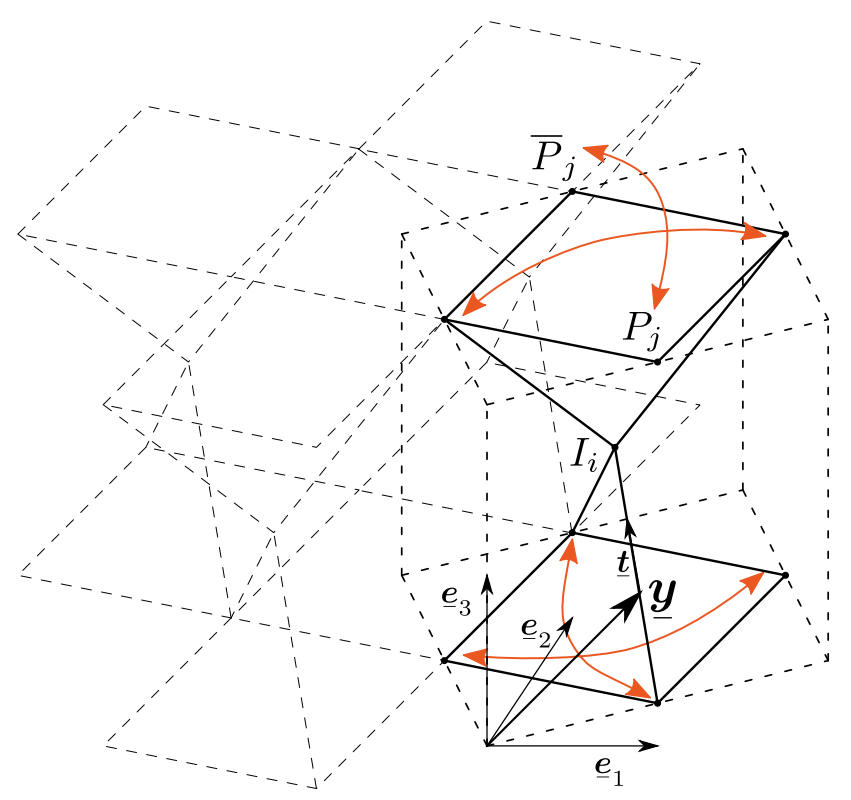

Figure 2: An example of unit-cell of a space frame with four pairs of periodicity nodes and one interior node.

Each beam is subjected to distributed forces $\underline{\boldsymbol{k}}$ and torques $\underline{\boldsymbol{l}}$. The corresponding equilibrium equations are:

$$
\left\{\begin{array}{l}
\underline{\boldsymbol{r}}^{\prime}+\underline{\boldsymbol{k}}=0 \\
\underline{\boldsymbol{m}}^{\prime}+\underline{\boldsymbol{t}} \times \underline{\boldsymbol{r}}+\underline{\boldsymbol{l}}=0
\end{array}\right.
$$

Where "' " denotes the derivative with respect to $s_{1}$. The constitutive equation writes as follows:

$$
\left\{\begin{array}{l}
\underline{\boldsymbol{r}}=\underset{\sim}{\boldsymbol{E}} \cdot\left(\underline{\boldsymbol{u}}^{\prime}+\underline{\boldsymbol{t}} \times \underline{\boldsymbol{\theta}}\right) \\
\underline{\boldsymbol{m}}=\underline{\boldsymbol{G}}^{\prime}
\end{array}\right.
$$

where $\underline{\boldsymbol{u}}$ is the beam displacement and $\underline{\boldsymbol{\theta}}$ its rotation. $\underset{\sim}{\boldsymbol{E}}$ and $\underset{\boldsymbol{G}}{\boldsymbol{a}}$ are the beam stiffness tensors. For instance, when there are two axis of symmetry for the section, these tensors write in the local reference frame of the beam $(\underline{\boldsymbol{t}}, \underline{\boldsymbol{n}}, \underline{\boldsymbol{b}})$, as:

$$
\begin{aligned}
\underset{\sim}{\boldsymbol{E}} & =\left(\begin{array}{ccc}
E \mathcal{S} & 0 & 0 \\
0 & G \mathcal{S}_{s, 2} & 0 \\
0 & 0 & G \mathcal{S}_{s, 3}
\end{array}\right) \\
\text { and } \quad \underset{\sim}{\boldsymbol{G}} & =\left(\begin{array}{ccc}
G \mathcal{J} & 0 & 0 \\
0 & E \mathcal{I}_{2} & 0 \\
0 & 0 & E \mathcal{I}_{3}
\end{array}\right)
\end{aligned}
$$

where $E$ is the Young modulus, $G$ the shear modulus of the constitutive material of the beam. $\mathcal{S}$ is the section area, $\mathcal{S}_{s, 2}$ and $\mathcal{S}_{s, 3}$ are the shear areas in each direction. $\mathcal{J}$ is the torsion constant, $\mathcal{I}_{2}=\int_{\mathcal{S}} s_{3}^{2} d \mathcal{S}$ and $\mathcal{I}_{3}=\int_{\mathcal{S}} s_{2}^{2} d \mathcal{S}$ are the second moments of inertia.

Kinematic and static constraints on the assembly. Since we assumed perfect connection, displacements and rotations must be continuous at interior nodes $I_{i}$ in all the following.

Moreover, local equilibrium at each interior node must be fulfilled:

$$
\forall j=1 \ldots n_{i}, \quad \sum_{k=1}^{n_{b j}} \epsilon_{k}{ }^{k} \underline{\boldsymbol{r}}\left(I_{j}\right)=0 \quad \text { and } \quad \sum_{k=1}^{n_{b j}} \epsilon_{k}{ }^{k} \boldsymbol{m}\left(I_{j}\right)=0
$$


where $n_{b j}$ is the number of beams connecting at node $I_{j},{ }^{k} \underline{r}$ denotes the resultant of beam $k$ connected to the node. Moreover, $\epsilon_{k}=+1$ if ${ }^{k} \underline{\boldsymbol{t}}$, the tangent vector of beam $k$, is directed toward the node and $\epsilon_{k}=-1$ if ${ }^{k} \underline{t}$ is directed away the node.

Additionally we define periodicity conditions as:

$$
\forall j=1 \ldots n_{p}, \quad \underline{\boldsymbol{\theta}}\left(P_{j}\right)=\underline{\boldsymbol{\theta}}\left(\bar{P}_{j}\right) \quad \text { and } \quad \underline{\boldsymbol{u}}\left(P_{j}\right)=\underline{\boldsymbol{u}}\left(\bar{P}_{j}\right)
$$

Elastic energy. The complementary elastic energy per unit surface stored inside the unit-cell will be used in the homogenization scheme. It is defined as the sum along $\Gamma$ of the beams stress energy density:

$$
w^{* \mathrm{int}}(\underline{\boldsymbol{r}}, \underline{\boldsymbol{m}})=\frac{1}{2 A} \int_{\Gamma}\left({ }^{T} \underline{\boldsymbol{r}} \cdot \underset{\sim}{\boldsymbol{E}^{-1}} \cdot \underline{\boldsymbol{r}}+{ }^{T} \boldsymbol{\boldsymbol { m }} \cdot \underline{\sim}^{-1} \cdot \underline{\boldsymbol{m}}\right) d \Gamma
$$

where $A$ is the in-plane area of the unit-cell

\subsubsection{Homogenization as Kirchhoff-Love plate}

In this section, we recast the Kirchhoff-Love auxiliary problem - originally defined for 3D continuum in a suitable form for the unit-cell we just introduced.

In the case of a 3D continuum, the Kirchhoff-Love auxiliary problem consists in applying the global displacement field

$$
\underline{\boldsymbol{U}}^{K L}=\underset{\sim}{\hat{\boldsymbol{e}}} \cdot \underline{\boldsymbol{y}}+y_{3} \underset{\sim}{\hat{\boldsymbol{\chi}}} \cdot \underline{\boldsymbol{y}}-\frac{1}{2}\left({ }^{T} \underline{\boldsymbol{y}} \cdot \underset{\sim}{\hat{\boldsymbol{\chi}}} \cdot \underline{\boldsymbol{y}}\right) \underline{\boldsymbol{e}}_{3}
$$

on average on the unit-cell, taking into account upper and lower free faces of the plate [14]. Here, we introduced the convenient notation:

$$
\begin{aligned}
\underset{\sim}{\hat{e}} & =\left(\begin{array}{ccc}
e_{11} & e_{12} & 0 \\
e_{21} & e_{22} & 0 \\
0 & 0 & 0
\end{array}\right) \\
\text { and } \quad \underset{\sim}{\hat{\chi}} & =\left(\begin{array}{ccc}
\chi_{11} & \chi_{12} & 0 \\
\chi_{21} & \chi_{22} & 0 \\
0 & 0 & 0
\end{array}\right)
\end{aligned}
$$

Then, the elastic energy stored in the unit-cell is computed for each pair of component of $\underset{\sim}{\boldsymbol{e}}$ and $\underset{\chi}{\chi}$ and yields the Kirchhoff-Love stiffness tensors $\underset{\approx}{\boldsymbol{A}}, \underset{\approx}{\boldsymbol{B}}, \underset{\approx}{\boldsymbol{D}}$.

In the present case, there is an additional displacement field which carries energy in the unit-cell: the 3D rotation field $\underline{\boldsymbol{\theta}}$. Rotations are related to the skew-symmetric part of the gradient of the displacement. The rotation pseudo-vector related to the skew symmetric part of $\underline{U}^{K L} \otimes \boldsymbol{\nabla}$ is :

$$
\underline{\Theta}^{K L}=\underset{\sim}{\omega} \cdot \underset{\sim}{\hat{\chi}} \cdot \underline{y}
$$

where $\underset{\sim}{\boldsymbol{\omega}}=\left(\underline{\boldsymbol{e}}_{2} \otimes \underline{\boldsymbol{e}}_{1}-\underline{\boldsymbol{e}}_{1} \otimes \underline{\boldsymbol{e}}_{2}\right)$ is the permutation operator. Thus we suggest to apply both $\underline{\boldsymbol{U}}^{K L}$ and $\underline{\boldsymbol{\Theta}}^{K L}$ on average on the unit-cell in the case of a space frame. This leads to the following Kirchhoff-Love auxiliary problem $\mathcal{P}^{K L}$ :

$$
\mathcal{P}^{K L}:\left\{\begin{array}{l}
\left(\underline{\boldsymbol{r}}^{K L}\right)^{\prime}=0 \text { on } \Gamma \\
\left(\underline{\boldsymbol{m}}^{K L}\right)^{\prime}+\underline{\boldsymbol{t}} \times \underline{\boldsymbol{r}}^{K L}=0 \text { on } \Gamma \\
\underline{\boldsymbol{r}}^{K L}=\underset{\sim}{\boldsymbol{E}} \cdot\left(\left(\underline{\boldsymbol{u}}^{K L}\right)^{\prime}+\underline{\boldsymbol{t}} \times \underline{\boldsymbol{\theta}}^{K L}\right) \text { on } \Gamma \\
\underline{\boldsymbol{m}}^{K L}=\boldsymbol{G} \cdot\left(\underline{\boldsymbol{\theta}}^{K L}\right)^{\prime} \text { on } \Gamma \\
\underline{\boldsymbol{u}}^{K L}=\underline{\boldsymbol{u}}^{\mathrm{per}}+\underline{\boldsymbol{U}}^{K L} \text { on } \Gamma \\
\underline{\boldsymbol{\theta}}^{K L}=\underline{\boldsymbol{\theta}}^{\text {per }}+\underline{\boldsymbol{\Theta}}^{K L} \text { on } \Gamma \\
\forall i=1 \ldots n_{i}, \quad \underline{\boldsymbol{u}}^{\text {per }} \text { and } \underline{\boldsymbol{\theta}}^{\text {per }} \text { continuous at node } I_{i} \\
\forall i=1 \ldots n_{i}, \quad \underline{\boldsymbol{r}}^{K L} \text { and } \underline{\boldsymbol{m}}^{K L} \text { equilibrated at node } I_{i} \\
\forall j=1 \ldots n_{p}, \quad \underline{\boldsymbol{u}}^{\text {per }}, \underline{\boldsymbol{\theta}}^{\text {per }}, \underline{\boldsymbol{r}}^{K L} \text { and } \underline{\boldsymbol{m}}^{K L} \text { periodic at node } P_{j}
\end{array}\right.
$$


where the subscript "per" refers to periodicity ( $\underline{\boldsymbol{u}}^{\mathrm{per}}$ and $\underline{\boldsymbol{\theta}}^{\mathrm{per}}$ are also named corrector fields).

NB: The free faces boundary condition is implicitly included in the present auxiliary problem. Actually, from one node to another, beams are free. In addition, no vertical displacement is prescribed at nodes. However, the displacement of one node belonging to the reference plane of the plate must be blocked in order to prevent rigid motion.

Solving the problem for each individual component of $\underset{\sim}{e}$ and $\underset{\sim}{\chi}$ leads to the stress localization tensors $r_{i \alpha \beta}^{e}, m_{i \alpha \beta}^{e}$ and $r_{i \alpha \beta}^{\chi}, m_{i \alpha \beta}^{\chi}$. The complete local stress field can be reconstructed by linear combination:

$$
\begin{gathered}
\underline{\boldsymbol{r}}^{K L}=\underline{\boldsymbol{r}}^{(e)}+\underline{\boldsymbol{r}}^{(\chi)}=\left(r_{i \alpha \beta}^{e} e_{\alpha \beta}\right)+\left(r_{i \alpha \beta}^{\chi} \chi_{\alpha \beta}\right)=\underline{\sim}^{e}: \underset{\sim}{\boldsymbol{e}}+\underline{\sim}^{\boldsymbol{r}}{ }^{\chi} \underset{\sim}{\chi} \\
\underline{\boldsymbol{m}}^{K L}=\underline{\boldsymbol{m}}^{(e)}+\underline{\boldsymbol{m}}^{(\chi)}=\left(m_{i \alpha \beta}^{e} e_{\alpha \beta}\right)+\left(m_{i \alpha \beta}^{\chi} \chi_{\alpha \beta}\right)={\underset{\sim}{\boldsymbol{m}}}^{e}: \underset{\sim}{\boldsymbol{e}}+{\underset{\sim}{\boldsymbol{m}}}^{\chi}: \underset{\sim}{\boldsymbol{\chi}}
\end{gathered}
$$

Kirchhoff-Love stiffness tensors are derived using Hill-Mandel principle. The Kirchhoff-Love plate energy density is identified to the energy stored in the unit-cell (Equation 10):

$$
w^{* \operatorname{int}}\left(\underline{\boldsymbol{r}}^{K L}, \underline{\boldsymbol{m}}^{K L}\right)=\frac{1}{2}(\underset{\sim}{\boldsymbol{e}}: \underset{\sim}{\boldsymbol{A}}: \underset{\sim}{\boldsymbol{e}}+2 \underset{\sim}{\boldsymbol{e}}: \underset{\sim}{\boldsymbol{B}}: \underset{\sim}{\boldsymbol{\chi}}+\underset{\sim}{\boldsymbol{\chi}}: \underset{\sim}{\boldsymbol{D}}: \underset{\sim}{\boldsymbol{\chi}})
$$

Kirchhoff-Love plate moduli are then evaluated as follows:

$$
\begin{aligned}
& \underset{\sim}{\boldsymbol{A}}=\frac{1}{A} \int_{\Gamma}\left({\underset{\sim}{\boldsymbol{r}}}^{e} \cdot \underset{\sim}{\boldsymbol{E}}{ }^{-1} \cdot{\underset{\sim}{\boldsymbol{r}}}^{e}+{ }^{T} \boldsymbol{\sim}^{e} \cdot \underset{\sim}{\boldsymbol{G}^{-1}} \cdot \underset{\sim}{\boldsymbol{m}}{ }^{e}\right) d \Gamma \\
& \underset{\approx}{\boldsymbol{B}}=\frac{1}{A} \int_{\Gamma}\left(\underline{\underline{\boldsymbol{r}}}^{\mathrm{T}} \cdot \underset{\sim}{\boldsymbol{E}}{ }^{-1} \cdot \underline{\underline{\boldsymbol{r}}}^{\chi}+{ }_{\simeq}^{T} \boldsymbol{\sim}^{e} \cdot \underline{\sim}^{-1} \cdot{\underset{\sim}{\boldsymbol{m}}}^{\chi}\right) d \Gamma \\
& \underset{\sim}{\boldsymbol{D}}=\frac{1}{A} \int_{\Gamma}\left({ }^{T} \underline{\underline{\boldsymbol{r}}}^{\chi} \cdot \underset{\sim}{\boldsymbol{E}}{ }^{-1} \cdot \underline{\sim}^{\chi}+{ }^{T} \boldsymbol{\sim}^{\chi} \cdot{\underset{\sim}{\boldsymbol{G}}}^{-1} \cdot \underset{\boldsymbol{m}^{\chi}}{\chi}\right) d \Gamma
\end{aligned}
$$

Finally, using the inverted constitutive equation,

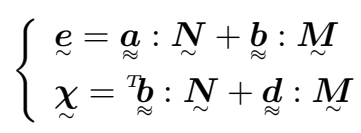

we get the following local stress field generated by uniform membrane stress $\underset{\sim}{N}$ and bending moment $M$ on the unit-cell:

$$
\begin{aligned}
& \underline{\boldsymbol{r}}^{(N)}={\underset{\underline{\boldsymbol{r}}}{ }}^{N}: \underset{\sim}{\boldsymbol{N}}=\left({\underset{\underline{\boldsymbol{r}}}{e}}^{e}: \underset{\sim}{\boldsymbol{a}}+{\underset{\underline{\boldsymbol{r}}}{ }}^{\chi}:{ }_{\sim}^{\mathrm{b}}\right): \underset{\sim}{\boldsymbol{N}} \\
& \underline{\boldsymbol{m}}^{(N)}={\underset{\sim}{\boldsymbol{m}}}^{N}: \underset{\sim}{\boldsymbol{N}}=\left(\underline{\sim}^{e}: \underset{\sim}{\boldsymbol{a}}+{\underset{\sim}{\boldsymbol{m}}}^{\chi}:{ }_{\sim}^{T} \boldsymbol{b}\right): \underset{\sim}{\boldsymbol{N}} \\
& \underline{\boldsymbol{r}}^{(M)}=\underline{\underline{\boldsymbol{r}}}^{M}: \underset{\sim}{M}=\left({\underset{\sim}{\boldsymbol{r}}}^{e}: \underset{\sim}{\boldsymbol{b}}+\underline{\sim}^{\chi}: \underset{\sim}{\boldsymbol{d}}\right): \underset{\sim}{\boldsymbol{M}} \\
& \underline{\boldsymbol{m}}^{(M)}={\underset{\sim}{\boldsymbol{m}}}^{M}: \underset{\sim}{\boldsymbol{M}}=\left({\underset{\sim}{\boldsymbol{m}}}^{e}: \underset{\sim}{\boldsymbol{b}}+{\underset{\sim}{\boldsymbol{m}}}^{\chi}: \underset{\sim}{\boldsymbol{d}}\right): \underset{\sim}{\boldsymbol{M}}
\end{aligned}
$$

\subsubsection{Homogenization as Bending-Gradient plate}

Once the Kirchhoff-Love homogenization is performed, it becomes possible to derive the BendingGradient localization field and constitutive equation. In [14], the homogenization scheme was suggested for 3D Cauchy continuum. However, in the present case, the unit-cell is constituted of structural elements. We suggest here a direct approach similar to the one used for deriving laminated plates localization fields [13].

Provided $\boldsymbol{M}$ is uniform in the unit-cell, the localization fields $\underline{\underline{r}}^{(M)}, \underline{\boldsymbol{m}}^{(M)}$ in (19) follow the equilibrium equations of Problem 14, Let us assume a macroscopic linear variation of $\underset{\sim}{M}$ through the unit-cell: $M_{\alpha \beta}^{*}=$ $M_{\alpha \beta}+R_{\alpha \beta \gamma} y_{\gamma}$ and define the Bending-Gradient localization field as:

$$
\underline{\boldsymbol{r}}^{B G}=\underline{\boldsymbol{r}}^{(N)}+\underline{\underline{r}}^{M}: \underline{\sim}^{*}+\underline{\boldsymbol{r}}^{(R)}
$$


and

$$
\underline{\boldsymbol{m}}^{B G}=\underline{\boldsymbol{m}}^{(N)}+\underline{\sim}^{M}: \underline{\sim}^{*}+\underline{\boldsymbol{m}}^{(R)}
$$

Injecting this field in Problem 14 leads to the definition of the Bending-Gradient auxiliary problem:

$$
\left\{\begin{array}{l}
\left(\underline{\boldsymbol{r}}^{(R)}\right)^{\prime}+\underline{\boldsymbol{k}}^{(R)}=0 \text { on } \Gamma \\
\left(\underline{\boldsymbol{m}}^{(R)}\right)^{\prime}+\underline{\boldsymbol{t}} \times \underline{\boldsymbol{r}}^{(R)}+\underline{\boldsymbol{l}}^{(R)}=0 \text { on } \Gamma \\
\underline{\boldsymbol{r}}^{(R)}=\underset{\sim}{\boldsymbol{E}} \cdot\left(\left(\underline{\boldsymbol{u}}^{(R)}\right)^{\prime}+\underline{\boldsymbol{t}} \times \underline{\boldsymbol{\theta}}^{(R)}\right) \text { on } \Gamma \\
\underline{\boldsymbol{m}}^{(R)}=\boldsymbol{G} \cdot\left(\underline{\boldsymbol{\theta}}^{(R)}\right)^{\prime} \text { on } \Gamma \\
\forall i=1 \ldots n_{i}, \quad \underline{\boldsymbol{u}}^{(R)} \text { and } \underline{\boldsymbol{\theta}}^{(R)} \text { continuous at node } I_{i} \\
\forall i=1 \ldots n_{i}, \quad \underline{\boldsymbol{r}}^{(R)} \text { and } \boldsymbol{\underline { m }}^{(R)} \text { equilibrated at node } I_{i} \\
\forall j=1 \ldots n_{p}, \quad \underline{\boldsymbol{u}}^{(\mathrm{R})}, \underline{\boldsymbol{\theta}}^{(\mathrm{R})}, \underline{\boldsymbol{r}}^{(R)} \text { and } \underline{\boldsymbol{m}}^{(R)} \text { periodic at node } P_{j}
\end{array}\right.
$$

where

$$
\begin{gathered}
\underline{\boldsymbol{k}}^{(R)}=\underline{\underline{\boldsymbol{r}}}^{M}: \underline{\boldsymbol{R}} \cdot \underline{\boldsymbol{t}}=\left(r_{i \alpha \beta}^{M} t_{\gamma} R_{\beta \alpha \gamma}\right) \\
\underline{\boldsymbol{l}}^{(R)}=\underline{\boldsymbol{m}}^{M}: \underline{\boldsymbol{R}} \cdot \underline{\boldsymbol{t}}=\left(m_{i \alpha \beta}^{M} t_{\gamma} R_{\beta \alpha \gamma}\right)
\end{gathered}
$$

are the distributed loadings generated by a uniform gradient of the bending moment $\boldsymbol{R}$.

Solving the problem for each individual component of $R_{\alpha \beta \gamma}$ leads to the localization stress fields $r_{i \gamma \beta \alpha}^{R}$, $m_{i \gamma \beta \alpha}^{R}$. The complete local stress field can be reconstructed by linear combination:

$$
\begin{aligned}
& \underline{\boldsymbol{r}}^{(R)}=\left(r_{i \gamma \beta \alpha}^{R} R_{\alpha \beta \gamma}\right)=\underline{\underline{r}}^{R} \vdots \underline{\boldsymbol{R}} \\
& \underline{\boldsymbol{m}}^{(R)}=\left(m_{i \gamma \beta \alpha}^{R} R_{\alpha \beta \gamma}\right)=\underline{\underline{m}}^{R}: \underline{\boldsymbol{R}}
\end{aligned}
$$

The generalized shear compliance is then evaluated as follows:

$$
\underset{\boldsymbol{f}}{\boldsymbol{f}}=\frac{1}{A} \int_{\Gamma}\left(\stackrel{\underline{T}}{\underline{\underline{T}}}^{R} \cdot{\underset{\sim}{\boldsymbol{E}}}^{-1} \cdot \underline{\underline{\boldsymbol{r}}}^{R}+\stackrel{T}{\underline{\boldsymbol{m}}}^{R} \cdot \boldsymbol{G}^{-1} \cdot \underline{\underline{\boldsymbol{m}}}^{R}\right) d \Gamma
$$

\section{Homogenization of a square lattice as a Bending-Gradient plate}

In order to illustrate this homogenization scheme, we consider a very simple structure: a square beam lattice (Figure 1). In this section, we derive the homogenized behavior of the lattice as a Bending-Gradient plate and also as a Reissner-Mindlin plate.

\subsection{The unit-cell}

Figure 3 shows the unit-cell of the lattice idealization. It is constituted of two beams ("h": horizontal, "v": vertical) which lie in the $\left(\underline{\boldsymbol{e}}_{1}, \underline{\boldsymbol{e}}_{2}\right)$ plane. Hence their local reference frames write as $\left(\underline{\boldsymbol{e}}_{1}, \underline{\boldsymbol{e}}_{2}, \underline{\boldsymbol{e}}_{3}\right)$ for beam "h" and $\left(\underline{e}_{2},-\underline{e}_{1}, \underline{e}_{3}\right)$ for beam "v". Both beams are identical with length $b$. We assume the beam section has two orthogonal axis of symmetry. One axis is $\underline{e}_{3}$ so that the beam stiffness tensors write as Equation 7 in the local reference frame.

\subsection{Kirchhoff-Love auxiliary problem}

Computation details of Kirchhoff-Love auxiliary problem are given in Appendix B. Here, we give directly the generalized stress field localization as function of $\underset{\sim}{N}$ and $\underset{\sim}{M}$ in the local reference frame of each beam:

$$
{ }^{\mathrm{h}} \underline{\boldsymbol{r}}^{K L}=\left(\begin{array}{c}
b N_{11} \\
b N_{12} \\
0
\end{array}\right)_{\mathrm{h}} \text { and } \quad{ }^{\mathrm{h}} \underline{\boldsymbol{m}}^{K L}=\left(\begin{array}{c}
-b M_{12} \\
b M_{11} \\
b N_{12}\left(s_{1}-\frac{b}{2}\right)
\end{array}\right)_{\mathrm{h}}
$$




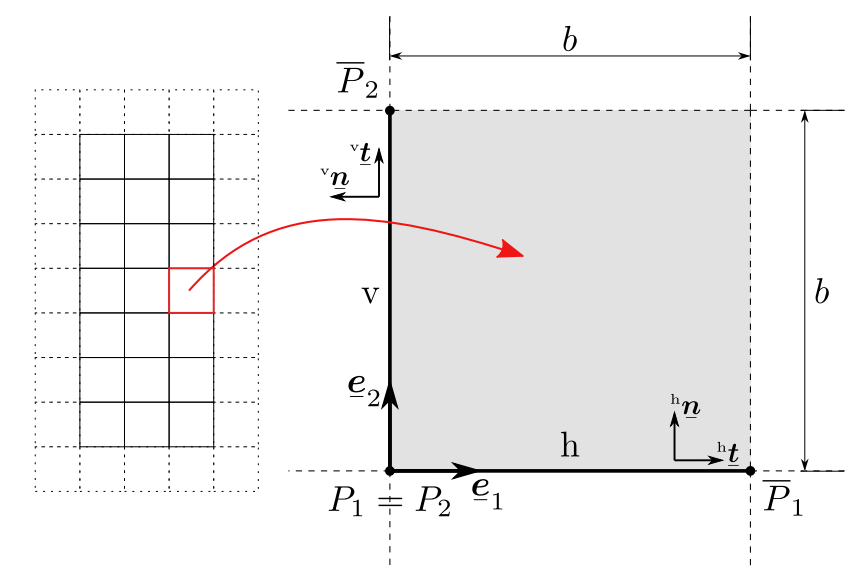

Figure 3: The beam lattice unit-cell

$$
{ }^{\mathrm{v}} \underline{\boldsymbol{v}}^{K L}=\left(\begin{array}{c}
b N_{22} \\
-b N_{12} \\
0
\end{array}\right)_{\mathrm{v}} \text { and } \quad{ }^{\mathrm{v}} \underline{\boldsymbol{m}}^{K L}=\left(\begin{array}{c}
b M_{12} \\
b M_{22} \\
-b N_{12}\left(s_{1}-\frac{b}{2}\right)
\end{array}\right)_{\mathrm{v}}
$$

This result is rather intuitive: membrane traction in Direction 1 generates only uniform traction of beam "h", as well as $N_{22}$ generates traction in beam "v". In plane shear $N_{12}$ puts both beams in non-uniform in-plane bending. Similarly, pure bending in Direction 1 generates pure out-of-plane bending of beam " $h$ " as well as $M_{22}$ generates pure out-of-plane bending in beam "v". Finally, plate torsion $M_{12}$ generates pure torsion in both beams.

$N B$ : All localization fields are continuous except the one generated by $N_{12}$. This is not incorrect, the generalized stress discontinuity being introduced by the node. Actually, the node is equilibrated.

The corresponding Kirchhoff-Love stiffness tensors are:

$$
[\underset{\sim}{\boldsymbol{A}}]=\left(\begin{array}{ccc}
\frac{E \mathcal{S}}{b} & 0 & 0 \\
0 & \frac{E \mathcal{S}}{b} & 0 \\
0 & 0 & \left(\frac{b}{G \mathcal{S}_{s, 2}}+\frac{b^{3}}{12 E \mathcal{I}_{3}}\right)^{-1}
\end{array}\right), \quad[\underset{\sim}{\boldsymbol{B}}]=0 \quad \text { and } \quad[\underset{\sim}{\boldsymbol{D}}]=\left(\begin{array}{ccc}
\frac{E \mathcal{I}_{2}}{b} & 0 & 0 \\
0 & \frac{E \mathcal{I}_{2}}{b} & 0 \\
0 & 0 & \frac{G \mathcal{J}}{b}
\end{array}\right)
$$

where we introduced Voigt notation $[\underset{\sim}{\boldsymbol{A}}]$ for fourth order tensors $\underset{\sim}{\boldsymbol{A}}$ as:

$$
[\underset{\approx}{\boldsymbol{A}}]=\left(\begin{array}{ccc}
A_{1111} & A_{2211} & \sqrt{2} A_{1211} \\
A_{2211} & A_{2222} & \sqrt{2} A_{1222} \\
\sqrt{2} A_{1211} & \sqrt{2} A_{1222} & 2 A_{1212}
\end{array}\right)
$$

$N B$ : the corresponding notation for $\underset{\sim}{N}$ and $\underset{\sim}{M}$ is:

$$
[\boldsymbol{N}]=\left(\begin{array}{c}
N_{11} \\
N_{22} \\
\sqrt{2} N_{12}
\end{array}\right)
$$

There is no coupling between $\underset{\sim}{\boldsymbol{N}}$ and $\underset{\sim}{\boldsymbol{M}}(\underset{\sim}{\boldsymbol{B}}=0)$ and there is no Poisson's effect $\left(A_{1122}=D_{1122}=0\right)$.

\subsection{Bending-Gradient and Reissner-Mindlin auxiliary problems}

\subsubsection{Bending-Gradient localization}

Once the Kirchhoff-Love auxiliary problem is solved, it is possible to derive the distributed loads of the Bending-Gradient auxiliary problem, $\underline{\boldsymbol{k}}^{(R)}$ and $\underline{\boldsymbol{l}}^{(R)}$ from Equation 23 The beam stress localization field 
related to $\underset{\sim}{M}$ is given in the local reference frame by:

$$
{ }^{\mathrm{h}} \underline{\boldsymbol{r}}^{(M)}={ }^{\mathrm{v}} \underline{\boldsymbol{r}}^{(M)}=\underline{\mathbf{0}} \quad, \quad{ }^{\mathrm{h}} \underline{\underline{\boldsymbol{m}}}^{(M)}=\left(\begin{array}{c}
-b M_{12} \\
b M_{11} \\
0
\end{array}\right)_{\mathrm{h}} \quad \text { and } \quad{ }^{\mathrm{v}} \underline{\boldsymbol{m}}^{(M)}=\left(\begin{array}{c}
b M_{12} \\
b M_{22} \\
0
\end{array}\right)_{\mathrm{v}}
$$

First, we have directly ${ }^{\mathrm{h}} \underline{\boldsymbol{k}}^{(R)}={ }^{\mathrm{v}} \underline{\boldsymbol{k}}^{(R)}=\underline{\mathbf{0}}$. Then, for beam "h" we have $\underline{\boldsymbol{R}} \cdot \underline{\boldsymbol{t}}=\left(R_{\alpha \beta 1}\right)$. Replacing $M_{\alpha \beta}$ by this in ${ }^{\mathrm{h}} \underline{\boldsymbol{m}}^{(M)}$ leads to:

$$
{ }^{\mathrm{h}} \underline{\underline{\boldsymbol{l}}}^{(R)}=\left(\begin{array}{c}
-b R_{121} \\
b R_{111} \\
0
\end{array}\right)
$$

Similarly for beam "v" we have:

$$
{ }^{\mathrm{v}} \underline{\underline{l}}^{(R)}=\left(\begin{array}{c}
b R_{122} \\
b R_{222} \\
0
\end{array}\right) \mathrm{v}
$$

The solution of Problem 22 is derived a very similar way as for Kirchhoff-Love auxiliary problem. It is not detailed here. The generalized stress localization in the local reference frame of each beam is:

$$
\begin{gathered}
{ }^{\mathrm{h}} \underline{\boldsymbol{r}}^{(R)}=\left(\begin{array}{c}
0 \\
0 \\
b\left(R_{111}+R_{122}\right)
\end{array}\right) \text { and } \quad{ }^{\mathrm{h}} \underline{\boldsymbol{m}}^{(R)}=\left(\begin{array}{c}
b R_{121}\left(s_{1}-\frac{b}{2}\right) \\
b R_{122}\left(s_{1}-\frac{b}{2}\right) \\
0
\end{array}\right)_{\mathrm{h}} \\
{ }^{\mathrm{v}} \underline{\boldsymbol{r}}^{(R)}=\left(\begin{array}{c}
0 \\
0 \\
b\left(R_{121}+R_{222}\right)
\end{array}\right) \text { and }{ }^{\mathrm{v}} \underline{\boldsymbol{m}}^{(R)}=\left(\begin{array}{c}
-b R_{122}\left(s_{1}-\frac{b}{2}\right) \\
b R_{121}\left(s_{1}-\frac{b}{2}\right) \\
0
\end{array}\right){ }_{\mathrm{V}}
\end{gathered}
$$

Let recall that $Q_{1}=R_{111}+R_{122}$ and $Q_{2}=R_{121}+R_{222}$. Thus the resultants ${ }^{\mathrm{h}} \underline{\boldsymbol{r}}^{(R)}$ and ${ }^{\mathrm{v}} \underline{\boldsymbol{r}}^{(R)}$ are directly function of the shear forces. However, the torsion part of shear forces $\left(R_{122}\right.$ and $\left.R_{121}\right)$ generates additional torsion and bending in ${ }^{\mathrm{h}} \underline{\boldsymbol{m}}^{(R)}$ and ${ }^{\mathrm{v}} \underline{\boldsymbol{m}}^{(R)}$. Thus, shear forces related to cylindrical bending and torsion do not generate the same local stress field.

\subsubsection{Reissner-Mindlin localization}

In order to compare in details the Bending-Gradient theory with Reissner-Mindlin theory, we also considered a Reissner-Mindlin homogenization scheme. We chose Cecchi and Sab [11] method for deriving a Reissner-Mindlin constitutive equation. Following the work from Whitney [4], Cecchi and Sab [11] assume the plate is under cylindrical bending for deriving local stress fields and a Reissner-Mindlin constitutive equation. The derivation is not detailed here. However, the related Reissner-Mindlin field localization is easily obtained, setting $R_{111}=Q_{1}, R_{222}=Q_{2}$ and $R_{121}=R_{122}=R_{112}=R_{221}=0$ in Equation 34

$$
\begin{aligned}
& { }^{\mathrm{h}} \underline{\boldsymbol{r}}^{(Q)}=\left(\begin{array}{c}
0 \\
0 \\
b Q_{1}
\end{array}\right)_{\mathrm{h}} \text { and } \quad{ }^{\mathrm{h}} \underline{\boldsymbol{m}}^{(Q)}=\underline{\mathbf{0}} \\
& { }^{\mathrm{v}} \underline{\boldsymbol{r}}^{(Q)}=\left(\begin{array}{c}
0 \\
0 \\
b Q_{2}
\end{array}\right) \text { and } \quad{ }^{\mathrm{v}} \underline{\boldsymbol{m}}^{(Q)}=\underline{\mathbf{0}}
\end{aligned}
$$

Thus, there is no more correction of $\boldsymbol{m}$. 


\subsubsection{Constitutive equations}

Bending-Gradient. The Bending-Gradient compliance is derived using Equation 26 and is:

$$
\underset{\boldsymbol{f}}{\boldsymbol{f}}=\frac{b}{G \mathcal{S}_{s, 3}} \underset{\sim}{\boldsymbol{i}} \cdot \underset{\sim}{\boldsymbol{i}}+\frac{b^{3}}{12}\left(\frac{1}{G \mathcal{J}}+\frac{1}{E \mathcal{I}_{2}}\right) \underline{\boldsymbol{t}}
$$

where

$$
[\underset{\sim}{\boldsymbol{i}} \cdot \underset{\sim}{\boldsymbol{i}}]=\left(\begin{array}{cccccc}
1 & 0 & 0 & 0 & 0 & 1 / \sqrt{2} \\
0 & 0 & 0 & 0 & 0 & 0 \\
0 & 0 & 1 / 2 & 0 & 1 / \sqrt{2} & 0 \\
0 & 0 & 0 & 0 & 0 & 0 \\
0 & 0 & 1 / \sqrt{2} & 0 & 1 & 0 \\
1 / \sqrt{2} & 0 & 0 & 0 & 0 & 1 / 2
\end{array}\right) \quad \text { and } \quad[\underline{\boldsymbol{t}}]=\left(\begin{array}{cccccc}
0 & 0 & 0 & 0 & 0 & 0 \\
0 & 0 & 0 & 0 & 0 & 0 \\
0 & 0 & 1 / 2 & 0 & 0 & 0 \\
0 & 0 & 0 & 0 & 0 & 0 \\
0 & 0 & 0 & 0 & 0 & 0 \\
0 & 0 & 0 & 0 & 0 & 1 / 2
\end{array}\right)
$$

in the main reference frame. We used the following Voigt notation (see [13] for full details):

$$
[\boldsymbol{f}]=\left(\begin{array}{cccccc}
f_{111111} & f_{111122} & \sqrt{2} f_{111121} & f_{111211} & f_{111222} & \sqrt{2} f_{111221} \\
f_{221111} & f_{221122} & \sqrt{2} f_{221121} & f_{221211} & f_{221222} & \sqrt{2} f_{221221} \\
\sqrt{2} f_{121111} & \sqrt{2} f_{121122} & 2 f_{121121} & \sqrt{2} f_{121211} & \sqrt{2} f_{121222} & 2 f_{121221} \\
f_{112111} & f_{112122} & \sqrt{2} f_{112121} & f_{112211} & f_{112222} & \sqrt{2} f_{112221} \\
f_{222111} & f_{222122} & \sqrt{2} f_{222121} & f_{222211} & f_{222222} & \sqrt{2} f_{222221} \\
\sqrt{2} f_{122111} & \sqrt{2} f_{122122} & 2 f_{122121} & \sqrt{2} f_{122211} & \sqrt{2} f_{122222} & 2 f_{122221}
\end{array}\right)
$$

In order to go further in the interpretation, we rewrite $f$ as:

$$
\underset{\sim}{\boldsymbol{f}}=\frac{b}{G \mathcal{S}_{s, 3}}(\underset{\sim}{\boldsymbol{i}} \cdot \underset{\sim}{\boldsymbol{i}}+\eta \underset{\boldsymbol{t}}{\stackrel{t}{\sim}})
$$

where

$$
\eta=\frac{b^{2} G \mathcal{S}_{s, 3}}{12}\left(\frac{1}{G \mathcal{J}}+\frac{1}{E \mathcal{I}_{2}}\right)
$$

Reissner-Mindlin. The Reissner-Mindlin shear force compliance is derived using Equation 26 again where the Reissner-Mindlin localization field (35) is plugged:

$$
f_{\alpha \beta}=\frac{b}{G \mathcal{S}_{s, 3}} \delta_{\alpha \beta}
$$

which is the surface average of individual beam shear force stiffness.

\subsection{Difference between Reissner-Mindlin and Bending-Gradient constitutive equation}

Setting $\eta=0$ in $\boldsymbol{f}$ leads exactly to the Reissner-Mindlin constitutive equation (411). Thus the additional term $\eta \underline{t}$ in $\boldsymbol{f}$ is a contribution purely related to the Bending-Gradient model. It reflects the additional torsion and bending in the beams when shear forces are not purely cylindrical in the main reference frame (eg: $Q_{1} \neq M_{11,1}$ ). In contrast to $\underset{\sim}{\boldsymbol{i}} \cdot \underset{\sim}{\boldsymbol{i}}$ which is an isotropic tensor for the Bending-Gradient constitutive equation (eg. invariant to in-plane rotations [13]), $\boldsymbol{t}$ is not isotropic which makes $\boldsymbol{f}$ sensitive to the orientation of the beam lattice.

Let us give some order of magnitude for $\eta$. For a circular cross section, we have: $\mathcal{S}_{s, 3}=\frac{9}{40} \pi d^{2}, \mathcal{I}_{2}=\frac{\pi d^{4}}{64}$ and $\mathcal{J}=2 \mathcal{I}_{2}$ where $d$ is the beam diameter. Thus:

$$
\eta=\frac{3 b^{2}}{5 d^{2}} \frac{2+\nu}{1+\nu} \approx \frac{b^{2}}{d^{2}}
$$


The ratio $b / d$ is typically larger than 4 (otherwise a 3D modelization of the unit-cell would be more suitable) and leads to $\eta$ typically larger than 10. Clearly, the contribution of $\eta \underline{t}$ to $\boldsymbol{f}$ is dominant.

This point is confirmed when deriving the relative distance between Bending-Gradient and ReissnerMindlin model introduced in Subsection 2.1

$$
\Delta^{R M / B G}=\frac{2 \sqrt{2}}{3} \frac{\eta}{\sqrt{8+(1+\eta)^{2}}}
$$

For large $\eta, \Delta^{R M / B G}=\frac{2 \sqrt{2}}{3} \approx 94 \%$.

Furthermore, for $\eta \gg 1$ the eigenvalues of $\boldsymbol{f}$ are approximately:

$$
\frac{b}{G \mathcal{S}_{s, 3}}\left(\frac{\eta}{2}, \frac{\eta}{2}, 1,1,0,0\right)
$$

Thus, depending on which eigenvalue of $\boldsymbol{f}$ is activated by the plate's configuration, the results might be quite different both in deflection and in local stress fields as will be illustrated in the next section.

\section{Cylindrical bending of a square beam lattice}

In order to give a clear demonstration of the additional warping effects included in the Bending-Gradient theory we compare Kirchhoff-Love, Reissner-Mindlin and Bending-Gradient models to the exact solution in cylindrical bending configuration where the lattice direction is rotated by $0^{\circ}$ and $45^{\circ}$ with respect to the bending direction.

\subsection{Lattice at $0^{\circ}$}

Figure 4 shows the lattice configuration when the bending direction is the same as the one of the lattice. It is bent in Direction $x_{1}$ along $2 n$ unit-cells and is infinitely periodic in Direction $x_{2}$. Longitudinal beams

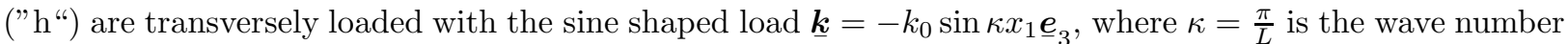
and $L$ is the span: $L=2 n b$.

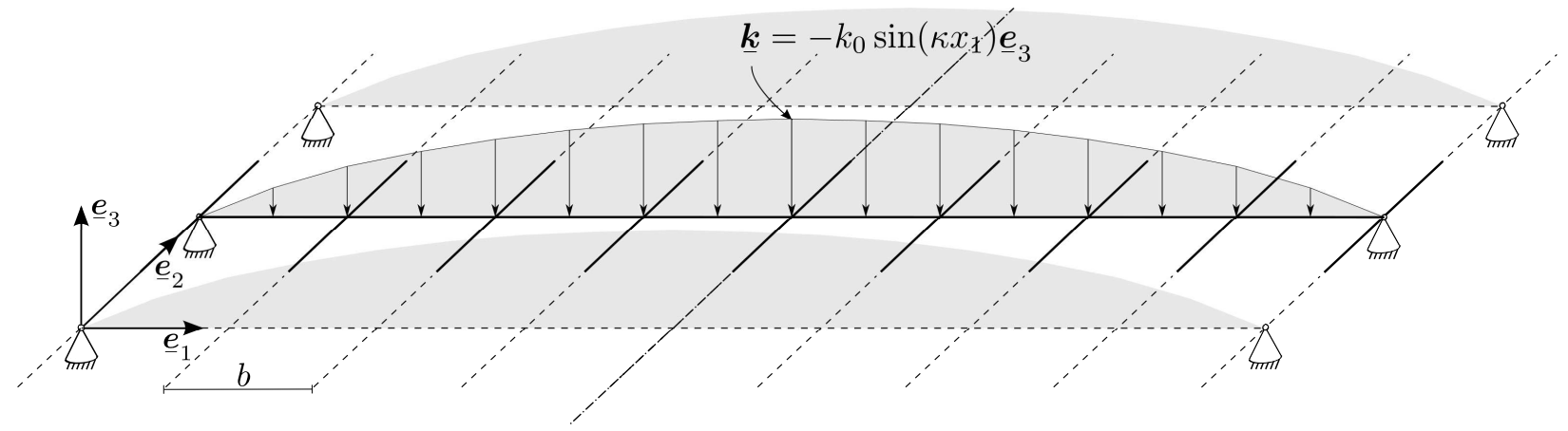

Figure 4: Beam lattice configuration at $0^{\circ}$ in cylindrical bending configuration

\subsubsection{Exact solution}

The exact solution for this configuration is straightforward. Transverse beams ("v") in Direction 2 are not loaded and longitudinal beams are simply supported with the transverse load $\underline{\boldsymbol{k}}$. The deflection along longitudinal beams is:

$$
{ }^{\mathrm{h}} u_{3}^{\mathrm{ex}}=-\frac{k_{0}}{\kappa^{4} E \mathcal{I}_{2}}\left(1+\frac{\pi^{2} E \mathcal{I}_{2}}{L^{2} G \mathcal{S}_{s, 3}}\right) \sin \kappa x_{1}
$$


the bending moment is:

$$
{ }^{\mathrm{h}} m_{2}^{\mathrm{ex}}=-\frac{k_{0}}{\kappa^{2}} \sin \kappa x_{1}
$$

and the shear force is:

$$
{ }^{\mathrm{h}} r_{3}^{\mathrm{ex}}=-\frac{k_{0}}{\kappa} \cos \kappa x_{1}
$$

\subsubsection{Homogenization approximations}

The plate surrogate for the configuration of the beam lattice given in Figure 4 is a simply supported plate under cylindrical bending. The plate distributed load is $\underline{\boldsymbol{p}}=k_{0} / b \sin \kappa x_{1} \underline{\boldsymbol{e}}_{3}$ in order to have the same loading per unit surface.

The boundary value problem is stated as follows. We assume invariance in $x_{2}$ direction. The plate is simply supported in $x_{1}=(0, L): U_{3}(0, L)=0, \underset{\sim}{M}(0, L)=0$ and loaded with $\underline{\boldsymbol{p}}$. We search for fields which are compatible (Equations (A.1) and (A.2)) and fulfill the constitutive equation (11). Computations are fully detailed in Appendix D.1. The Bending-Gradient and the Reissner-Mindlin solutions are both strictly identical to the exact solution. The Kirchhoff-Love approximation yields a less detailed result. The macroscopic deflection is:

$$
U_{3}^{K L}=-\frac{k_{0}}{\kappa^{4} E \mathcal{I}_{2}} \sin \kappa x_{1}
$$

There is no localization related to shear forces and then:

$$
\begin{gathered}
{ }^{\mathrm{h}} r_{3}^{K L}=0 \\
{ }^{\mathrm{h}} m_{2}^{K L}=-\frac{k_{0}}{\kappa^{2}} \sin \kappa x_{1}
\end{gathered}
$$

As expected, the Kirchhoff-Love homogenization scheme underestimates the deflection because it does not take into account second order effects related to $\boldsymbol{R}$. Additionally it does not capture local stress generated by shear forces. However, it is common practice for laminated plates to use the $3 \mathrm{D}$ equilibrium equation integrated through the thickness in order to derive this field. Here, we can use the microstructure's beam equilibrium equation (5) for this: plugging $m_{2}^{K L}$ into equation (5) gives the correct shear force in the beam.

\subsection{Lattice at $45^{\circ}$}

Now we simply rotate the lattice of $45^{\circ}$ with respect to the bending direction. Figure 5 shows the lattice in this new configuration. It is bent in Direction $x_{1}$ along $2 n$ diamond shaped unit-cells and is infinitely periodic in direction $x_{2}$. The beams are transversely loaded with the sine shaped load $\underline{k}=-k_{0} \sin \kappa x_{1} \underline{e}_{3}$. Now the span is: $L=2 \sqrt{2} n b$ (Figure 6 .

There are two sets of beams. The first set "+" is numbered $1 . .2 n$ and is rotated by $+\pi / 4$ in $\left(\underline{e}_{1}, \underline{e}_{2}\right)$ plane and the second set " - " is rotated by $-\pi / 4$. It is symmetric to set + with respect to any $\left(A^{j}, \underline{e}_{1}, \underline{e}_{3}\right)$ plane. Hence the focus is on set + in the following.

Beams are perfectly connected at nodes $A_{j}$ located at $x_{1}=x^{j}=j b / \sqrt{2}$. Simple support condition occurs at node $A^{0}$ and $A^{4 n}$ : both beams are perfectly connected together, however the node is free to rotate with respect to the support.

\subsubsection{Exact solution}

The derivation of the exact solution of this problem, though cumbersome, does not raise any difficulty. It is detailed in Appendix C The results are summarized here for the set of beams "+". The generalized stresses in the local reference frame of the beam for $x^{j}<x_{1}<x^{j+1}$ are:

$$
{ }^{+} \underline{\boldsymbol{e x}}^{\mathrm{ex}}=-\sqrt{2} \frac{k_{0}}{\kappa} \cos \kappa x_{1} \underline{\boldsymbol{e}}_{3}
$$




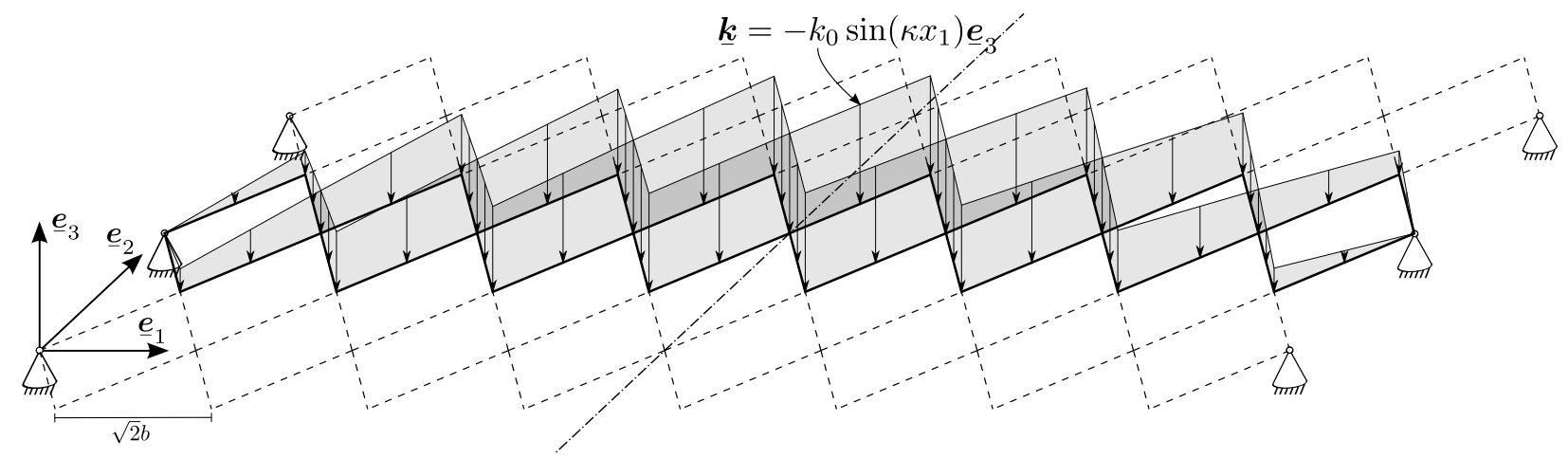

Figure 5: Beam lattice configuration at $45^{\circ}$ in cylindrical bending configuration

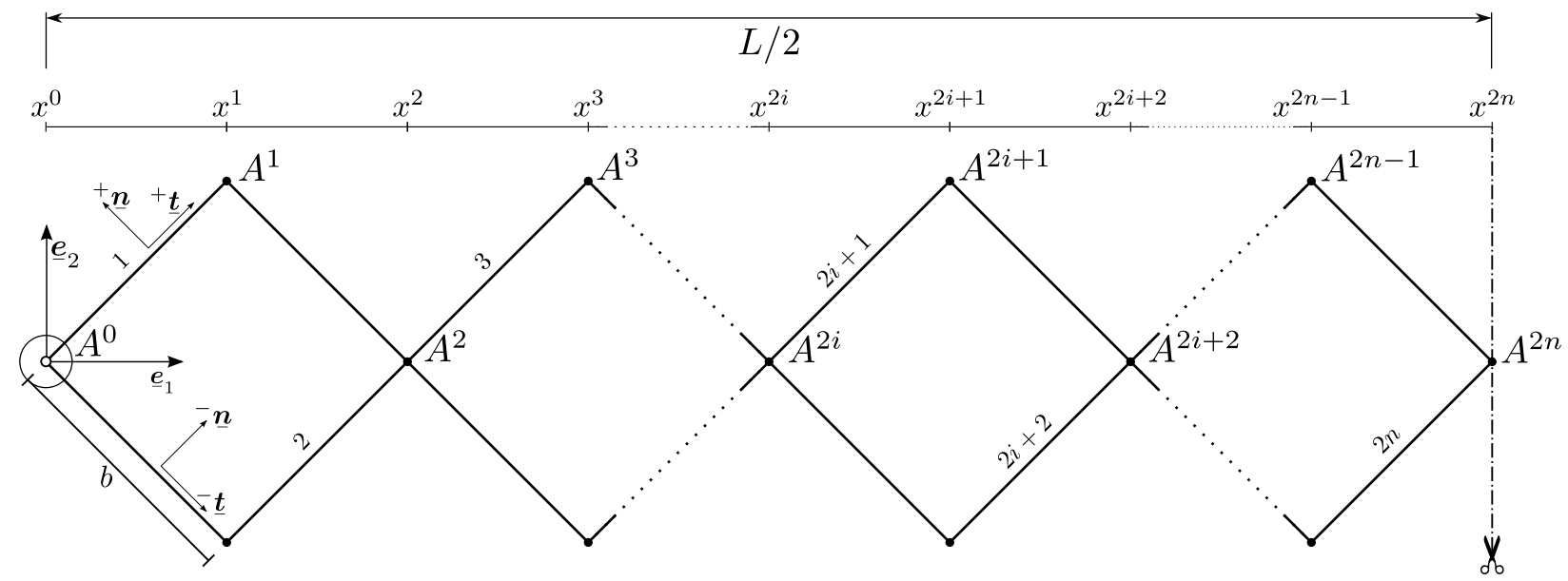

Figure 6: Top view of the beam lattice at $45^{\circ}$ and detailed parameters (only half a span is shown) 


$$
{ }^{+} \underline{\boldsymbol{m}}^{\mathrm{ex}}=-m^{*}\left(\begin{array}{c}
\frac{\alpha}{1+\alpha} \frac{\cos _{j}-\cos _{j+1}}{2 \sqrt{3} \gamma} \\
\sin \kappa x_{1}+\frac{\alpha}{1+\alpha} \frac{\cos _{j+1}-\cos _{j}}{2 \sqrt{3} \gamma} \\
0
\end{array}\right)_{+}
$$

where $\cos _{j}=\cos \kappa x^{j}, \alpha=\frac{G \mathcal{J}}{E \mathcal{I}_{2}}$ is the ratio between torsion and bending of the beam, $\gamma^{2}=\frac{\kappa^{2} b^{2}}{24}$ is the unit-cell size versus span ratio and $m^{*}=\frac{2 k_{0}}{\kappa^{2}}$ is the maximum bending moment in the beams. The resultant ${ }^{+} \underline{\boldsymbol{r}}^{\mathrm{ex}}$ is rather similar to the $0^{\circ}$ case. However, with ${ }^{+} \underline{\boldsymbol{m}}^{\mathrm{ex}}$, there is an additional uniform torsion in each beam.

The deflection along beams "+" for $x^{j}<x_{1}<x^{j+1}$ is:

$$
\begin{aligned}
+u_{3}^{\mathrm{ex}}=- & U^{K L}\left(\left(1+\frac{\gamma^{2}(1+\alpha)}{\eta \alpha}\right)(1+\alpha) \sin \kappa x_{1}\right. \\
& \left.\quad-\sqrt{3} \gamma \alpha\left(\cos _{j+1}\left(\bar{x}_{1}-j\right)^{2}-\cos _{j}\left(\bar{x}_{1}-j-1\right)^{2}+\frac{\cos _{j}-\cos _{j+1}}{1-\cos _{1}}\right)\right)
\end{aligned}
$$

where $\bar{x}_{1}=\sqrt{2} x_{1} / b$ is the normalized coordinate and $U^{K L}=\frac{4 k_{0}}{\kappa^{4} E \mathcal{I}_{2}(1+\alpha)}$ is Kirchhoff-Love maximum deflection.

\subsubsection{Homogenization approximations}

The plate surrogate for the configuration in Figure 5 is a simply supported plate under cylindrical bending where the distributed load is now $\underline{\boldsymbol{p}}=\frac{2 k_{0}}{b} \sin \kappa x_{1} \underline{\boldsymbol{e}}_{3}$ in order to have the same loading per unit surface. The boundary value problem is exactly the same as in the $0^{\circ}$ case. Hence the approximation is derived following a similar procedure. However one have to take into account the rotation of the lattice. Full details are given in Appendix D.2

Deflection. The macroscopic deflection for the Bending-Gradient is:

$$
U_{3}^{B G}=-U^{K L}(1+\frac{\gamma^{2}}{\alpha} \underbrace{\left(\frac{(1+\alpha)^{2}}{\eta}\right.}_{R M} \underbrace{\left.+\frac{\alpha^{2}}{1+\gamma^{2}}\right)}_{B G}) \sin \kappa x_{1}
$$

Removing underbraced terms with "BG" leads to Reissner-Mindlin approximation and removing both "BG" and "RM" terms leads to Kirchhoff-Love deflection.

For most sections $\alpha \approx 1$ and $\gamma$ is small. Since $\eta$ is typically larger than 10 , the "BG" term in Equation 53 is large compared to the "RM" one. Thus, the Reissner-Mindlin approach underestimates the actual second order deflection. This is confirmed when plotting the deflection error, $\frac{U_{3}-u_{3}^{\text {ex }}}{U^{K L}}$ along the span (Figure 7 ). Beams have the same circular cross section as in Section 3.3. The Poisson's coefficient is 0.3 and the ratio between the cell-size and the section diameter is $b / d=5$, which leads to $\eta \simeq 26$. The span is made of four unit-cells $\left(n=2,0<\bar{x}_{1}<4 n\right)$. Only half the span is plotted versus the normalized coordinate $\bar{x}_{1}=\sqrt{2} x_{1} / b$.

Globally, the errors remain small compared to the exact solution. As expected Kirchhoff-Love approximation underestimates the deflection. However, the correction given by Reissner-Mindlin approximation is not large enough. Actually, only the Bending-Gradient is able to properly capture all corrections related to second order effects.

Local fields. Local stress fields in the beams with the Bending-Gradient approximation write as follow:

$$
{ }^{+} \underline{\boldsymbol{r}}^{B G}=\underbrace{-\frac{\sqrt{2} k_{0}}{\kappa} \cos \kappa x_{1} \underline{\boldsymbol{e}}_{3}}_{R M}
$$




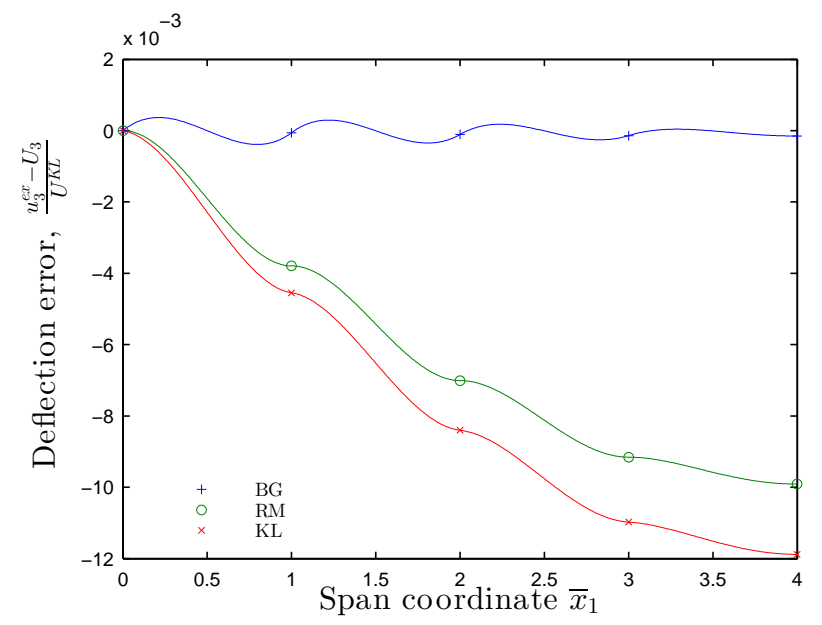

Figure 7: Macroscopic deflection error along the span for a 4 unit-cell lattice (only half the span is plotted)

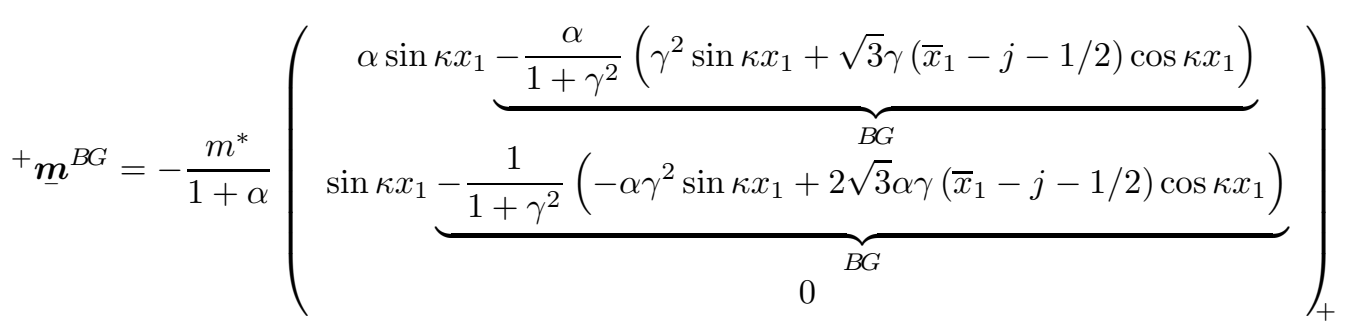

where the same convention as in Equation 53 is used.

Exactly as in the $0^{\circ}$ case (Section 4.1), Kirchhoff-Love approximation does not capture the shear forces $r_{3}$ in the beams. In addition, using the beam equilibrium for recovering the effect shear forces as we did in Subsubsection (4.1.2) leads to ${ }^{+} \underline{\boldsymbol{r}}=-\frac{1}{1+\alpha} \frac{\sqrt{2} k_{0}}{\kappa} \cos \kappa x_{1} \underline{\boldsymbol{e}}_{3}$ which underestimates the actual shear force. This fact was already pointed out in [13] when dealing with laminated plates. The FE simulation using integration through the thickness for deriving transverse shear stress distribution was leading to un-balanced fields when the direction of orthotropy was not the same as the direction of bending. Considering now the Reissner-Mindlin approximation, there is an additional correction for $\underline{\boldsymbol{m}}^{B G}$ which is only carried by the Bending-Gradient solution.

In order to have a closer look at this contribution, the bending moment $m_{2}$ and the torsion $m_{1}$ along beams "+" are plotted respectively in Figures 8 and 9 for the exact solution and homogenization approximations. Kirchhoff-Love and Reissner-Mindlin solutions are identical and plotted as the same continuous curve which follows the exact solution on average. By contrast, the Bending-Gradient approximation follows closely the exact solution. Especially, it captures the change of sign of the bending moment $m_{2}$ close to the support. In case the lattice is made of concrete, this kind detail is useful for an efficient design. This is not included in the other approximations.

The errors $\frac{m_{1}-m_{1}^{\text {ex }}}{m^{*}}$ and $\frac{m_{2}-m_{2}^{\text {ex }}}{m^{*}}$ are also plotted in Figures 10 and 11. It shows that the maximum error is $1 \%$ for the Bending-Gradient and $9 \%$ for the other approaches.

\section{Discussion}

In the previous section, three homogenization approximations of the cylindrical bending of a beam lattice were derived. The first one, leading to a Kirchhoff-Love plate model is also known to be the first order of the asymptotic expansion [1, 2]. This approach enables to have a first order estimate of the macroscopic deflection as well as local stress fields. Except with sandwich panels and laminated plates, in most application 


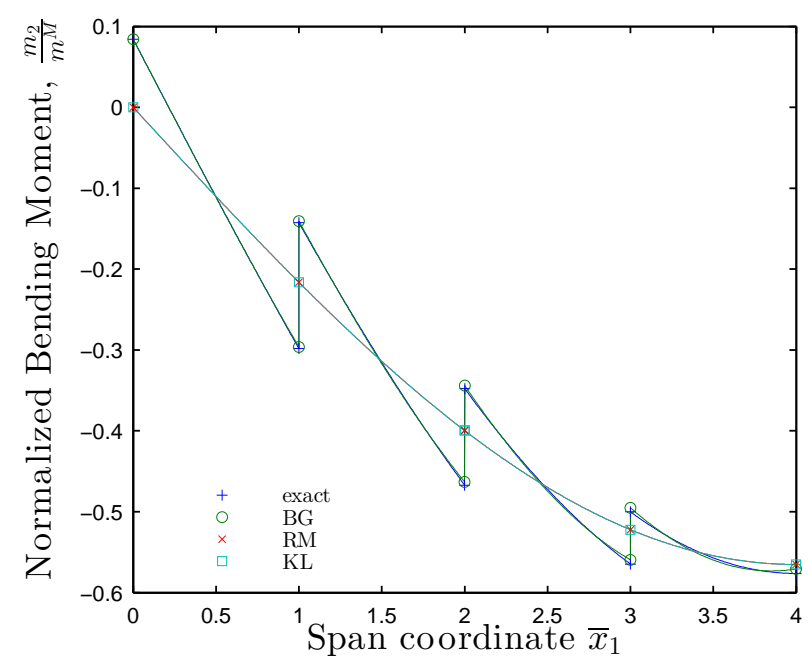

Figure 8: Bending moment $m_{2}$ along Beams "+" for a 4 unit-cell lattice (only half the span is plotted)

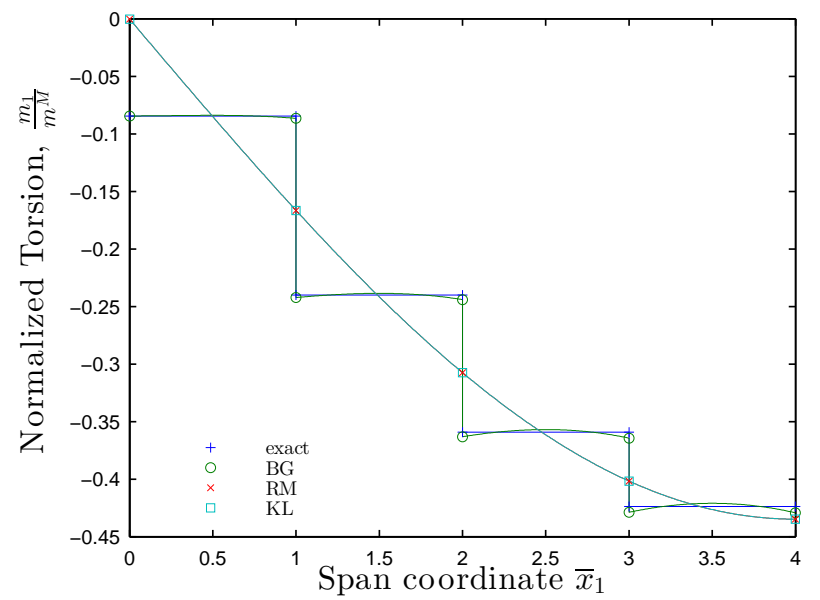

Figure 9: Torsion $m_{1}$ along Beams "+" for a 4 unit-cell lattice (only half the span is plotted) 


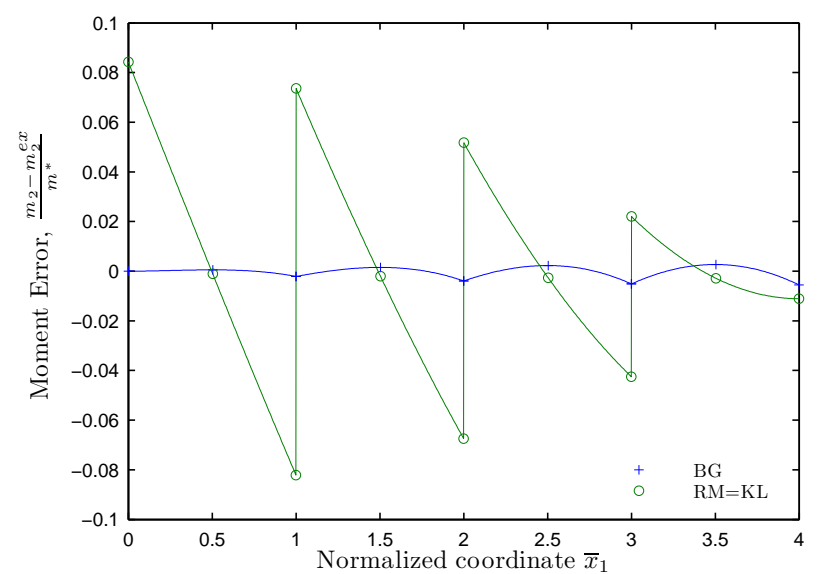

Figure 10: Bending moment error along Beams "+" for a 4 unit-cell lattice (only half the span is plotted)

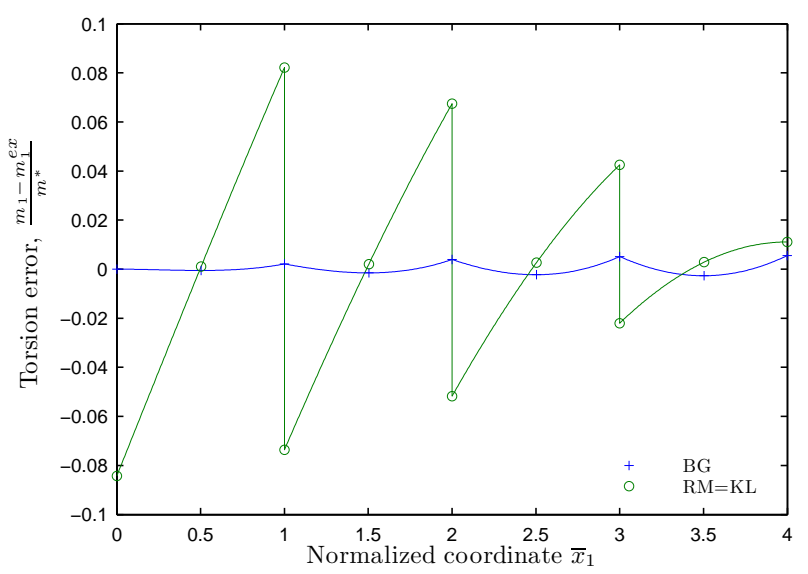

Figure 11: Torsion error along Beams "+" for a 4 unit-cell lattice (only half the span is plotted) 
the first order deflection is accurate enough. This is also the case in this paper (the error is already smaller than $10^{-3}$ for a span of few unit-cells). However, this approach does not capture the local effect of shear forces on the microstructure and the common practice for deriving the actual effect of transverse shear appears clearly as not reliable in more general configurations.

The Reissner-Mindlin homogenization approach used in this paper clearly misses some part of their effect. This is because of the assumption of cylindrical bending which is made in most approach for deriving a Reissner-Mindlin-like constitutive equation. This assumption enforces $Q_{1}=M_{11,1}$ and precludes any possible effect of the other derivatives of the bending moment on the local stress on the microstructure.

In the present case, the Bending-Gradient approximation captures all second order effects and gives a very satisfying description of both the deflection and the local stress fields. Especially, when rotating the lattice, this accuracy is preserved. Moreover, it brought out two scaling parameters between the first order deflection and the second order deflection. The first one is related to shear forces in the beams and is the same as the one in the Reissner-Mindlin approach. It is proportional to $\gamma^{2} / \eta$, namely $(d / L)^{2}$. The second one is related to the second order torsion/bending of the beams and is proportional to $\gamma^{2}$, namely $(b / L)^{2}$. Thus the latter effect is dominant at second order provided it is activated by the plate configuration. This distinction between scaling parameters is completely discarded when using a Reissner-Mindlin approach.

Our approach still presents some limitations. First, in the present case, we chose supports conditions for the lattice so that edge effects remain limited. It is well known in homogenization that these effects might be not negligible in some configurations. This point is far out of the scope of this paper and raises the question - common to all models - regarding the mechanical meaning of plate boundary conditions. Second, the local load distribution on the microstructure was arbitrarily chosen. At first order, this choice does not affect the fields localization (Kirchhoff-Love case). However, different load distributions could generate different localization of stress fields when looking for a higher order approximation (see [24] for instance).

Finally, in the present paper, the plate model was solved in a simple, closed-form solution. This raises the question regarding the finite element implementation of the Bending-Gradient theory. Actually, it is very close to the classical Reissner-Mindlin model (with its well-known limitations). The most straightforward implementation is to add the four $\mathcal{C}^{0}$ generalized rotation fields which are coupled to Reissner-Mindlin FEM formulation. This way the computational burden remains perfectly acceptable.

\section{Conclusion}

In this paper, the Bending-Gradient homogenization scheme was extended to space frames. Its application to a square beam lattice led to closed form solutions of the auxiliary problems. It also revealed that this microstructure cannot be properly modeled with Reissner-Mindlin theory. This observation was confirmed when comparing homogenization approaches with the exact solution of the cylindrical bending of the lattice. Only the Bending-Gradient theory is able to capture second order effects both in macroscopic deflection and in local fields.

Many other periodic structures might not be correctly modeled with Reissner-Mindlin theory such as concrete waffle slabs, orthotropic decks or composite floor systems and requires more accurate approaches such as the Bending-Gradient model. Hence, let us point out that it is perfectly possible to model a microstructure which is a mix between 3D, shell or beam elements following the direct derivation of the Bending-Gradient auxiliary problem suggested in the present paper and that other patterns are currently under investigation.

One can ask also if the Bending-Gradient theory can be easily extended to more random microstructures. The answer is actually the same as for case of a 3D random medium: it might be possible to establish bounds and argue on the statistical distribution of the microstructure. However, there is no clear argument for expecting that the Bending-Gradient would turn back to a Reissner-Mindlin model.

Finally, provided the limitations pointed out in Section [5 are kept in mind, the Bending-Gradient plate theory is considered by the authors as a sound extension of the Reissner-Mindlin theory to the case of heterogeneous plates. More precisely, it is an attempt to concentrate most of the results given by the second order of the asymptotic expansion [10] into a model which keeps most aspects of Reissner-Mindlin model, well understood by engineers. 


\section{Appendix A. Compatible fields for the Bending-Gradient theory}

Equilibrium equations and boundary conditions involving stress fields are gathered in the set of statically compatible fields:

$$
\left\{\begin{array}{l}
\underset{\sim}{\boldsymbol{N}} \cdot \underline{\boldsymbol{\nabla}}=\underline{\mathbf{0}} \text { on } \omega \\
\underset{\sim}{\boldsymbol{M}} \otimes \underline{\boldsymbol{\nabla}}-\underline{\boldsymbol{R}}=\mathbf{0} \text { on } \omega \\
(\underset{\sim}{\boldsymbol{i}}: \underline{\boldsymbol{R}}) \cdot \underline{\boldsymbol{\nabla}}=-p_{3} \text { on } \omega \\
\boldsymbol{\sim} \cdot \underline{\boldsymbol{n}}=\underline{\boldsymbol{V}}^{d} \text { on } \partial \omega^{s} \\
\underset{\sim}{\boldsymbol{M}}=\underline{\sim}^{d} \text { on } \partial \omega^{s} \\
(\underset{\sim}{\boldsymbol{i}}: \underline{\boldsymbol{R}}) \cdot \underline{\boldsymbol{n}}=V_{3}^{d} \text { on } \partial \omega^{s}
\end{array}\right.
$$

where the derivation operator $\underline{\boldsymbol{\nabla}}$ is also formally represented as a vector: $\underset{\boldsymbol{a}}{\boldsymbol{\theta}} \cdot \underline{\boldsymbol{\nabla}}=a_{\alpha \beta} \nabla_{\beta}=a_{\alpha \beta, \beta}$ is the divergence and $\underset{\sim}{\boldsymbol{a}} \otimes \boldsymbol{\nabla}=a_{\alpha \beta} \nabla_{\gamma}=a_{\alpha \beta, \gamma}$ is the gradient. $\partial \omega^{s}$ is the portion of edge on which static boundary conditions apply and $\underline{\boldsymbol{n}}$ the related outer normal: $\underline{\boldsymbol{V}}^{d}=\left(V_{i}^{d}\right)$ is the force per unit length and $\boldsymbol{M}^{d}$ the full bending moment enforced on the edge. The plate is loaded exclusively with the out-of-plane distributed force $\boldsymbol{p}=p_{3} \underline{\boldsymbol{e}}_{3}$. This set of equations is almost identical to Reissner-Mindlin equations where shear forces have been replaced by the bending gradient $\boldsymbol{R}$.

The compatibility conditions and boundary conditions for strain fields are gathered in the set of kinematically compatible fields:

$$
\left\{\begin{array}{l}
\underset{\sim}{\boldsymbol{e}}=\underline{\boldsymbol{U}} \otimes^{s} \boldsymbol{\nabla} \text { on } \omega \\
\underset{\boldsymbol{\chi}}{\boldsymbol{\chi}}=\underline{\mathbf{\Phi}} \cdot \underline{\boldsymbol{\nabla}} \text { on } \omega \\
\underline{\boldsymbol{\Gamma}}=\underline{\mathbf{\Phi}}+\underset{\sim}{\boldsymbol{i}} \cdot \underline{\nabla} U_{3} \text { on } \omega \\
\boldsymbol{\Phi} \cdot \boldsymbol{n}=\underline{\sim}^{d} \text { on } \partial \omega^{k} \\
\underline{\boldsymbol{U}}=\underline{\boldsymbol{U}}^{d} \text { on } \partial \omega^{k}
\end{array}\right.
$$

where $\underline{U}=\left(U_{i}\right)$ is the average through the thickness of the plate 3D displacement and $\boldsymbol{\Phi}=\left(\Phi_{\alpha \beta \gamma}\right)$ is the generalized rotation. $\boldsymbol{\Gamma}$ and $\Phi$ are 2D-third-order tensors with the following symmetry: $\Phi_{\alpha \beta \gamma}=\Phi_{\beta \alpha \gamma}$. Moreover, $\partial \omega^{k}$ is the portion of edge on which kinematic boundary conditions apply: $\underline{U}^{d}=\left(U_{i}^{d}\right)$ is a given displacement and ${\underset{\sim}{\boldsymbol{H}}}^{d}=\left(H_{\alpha \beta}^{d}\right)$ is a symmetric second-order tensor related to a forced rotation on the edge. These fields are almost identical to Reissner-Mindlin kinematically compatible fields where the rotation vector is replaced by the generalized rotation $\underline{\Phi}$. Assuming $\underset{\boldsymbol{\Phi}}{\boldsymbol{\Phi}} \underset{\sim}{\boldsymbol{i}} \cdot \boldsymbol{\varphi}$ in (A.2), where $\boldsymbol{\varphi}$ is a vector representing rotations leads to Reissner-Mindlin-like kinematics: $\underset{\boldsymbol{\Gamma}}{=} \underset{\sim}{\boldsymbol{i}} \cdot\left(\underline{\varphi}+\underline{\boldsymbol{\nabla}} U_{3}\right)=\underset{\sim}{\boldsymbol{i}} \cdot \underline{\gamma}$ and $\underset{\sim}{\boldsymbol{\chi}}=\underline{\varphi} \otimes^{s} \underline{\nabla}$. Thus in the general case, Reissner-Mindlin kinematics can be interpreted as a restriction of $\Phi$ to $\underset{\sim}{\boldsymbol{i}} \cdot \underline{\varphi}$.

\section{Appendix B. Computation details for Kirchhoff-Love homogenization}

In this section we detail the application of the Kirchhoff-Love auxiliary problem to the unit-cell of the lattice given in Figure 3. For convenience, the "KL" superscript for the solution is dropped in this section.

Node equilibrium. The equilibrium of node $P_{1}$ leads to:

$$
\left\{\begin{array}{l}
\Delta^{\mathrm{h}} \underline{\boldsymbol{r}}+\Delta^{\mathrm{v}} \underline{\boldsymbol{r}}=\underline{\mathbf{0}} \\
\Delta^{\mathrm{h}} \underline{\boldsymbol{m}}+\Delta^{\mathrm{v}} \underline{\boldsymbol{m}}=\underline{\mathbf{0}}
\end{array}\right.
$$

where $\Delta \underline{\boldsymbol{m}}=\boldsymbol{\boldsymbol { m }}\left(s_{1}=0\right)-\boldsymbol{m}\left(s_{1}=b\right)$ and the left superscript denotes the beam (see Figure 3). 
Beam equilibrium. Equilibrium equations (5) applied to beam "h" lead to uniform resultant along the beam:

$$
{ }^{\mathrm{h}} \underline{\underline{r}}={ }^{\mathrm{h}} \underline{\underline{0}}^{0}
$$

and linear bending moment:

$$
{ }^{\mathrm{h}} \underline{\boldsymbol{m}}=-\underline{\boldsymbol{e}}_{1} \times{ }^{\mathrm{h}} \underline{\boldsymbol{r}}\left(s_{1}-b / 2\right)+{ }^{\mathrm{h}} \underline{\boldsymbol{m}}^{\frac{b}{2}}
$$

where ${ }^{\mathrm{h}} \underline{\underline{b}}^{\frac{b}{2}}$ is the bending moment taken at $s_{1}=b / 2$. Similar results occur for beam "v".

We focus now on the derivation of ${ }^{\mathrm{h}} \underline{\underline{0}}^{0}$ and ${ }^{\mathrm{h}} \underline{\boldsymbol{m}}^{\frac{b}{2}}$ which fully determine the stress state of beam "h". Inserting this in node equilibrium B.1 leads to:

$$
\underline{\boldsymbol{e}}_{1} \times{ }^{\mathrm{h}} \underline{\boldsymbol{r}}^{0}+\underline{\boldsymbol{e}}_{2} \times{ }^{\mathrm{v}} \underline{\boldsymbol{r}}^{0}=0
$$

Taking the cross product with $\underline{e}_{1}$ enforces: ${ }^{\mathrm{h}} r_{2}^{0}=-{ }^{\mathrm{v}} r_{2}^{0}$ and ${ }^{\mathrm{h}} r_{3}^{0}={ }^{\mathrm{v}} r_{3}^{0}=0$, where the components are taken in the local reference frame of each beam.

Beam displacement. The constitutive equation 6 related to rotations leads to:

$$
{ }^{\mathrm{h}} \boldsymbol{G} \cdot\left({ }^{\mathrm{h}} \boldsymbol{\theta}-{ }^{\mathrm{h}} \underline{\boldsymbol{\theta}}^{0}\right)=\underline{e}_{1} \times{ }^{\mathrm{h}} \underline{\boldsymbol{r}}^{0}\left(s_{1}-b\right) \frac{s_{1}}{2}+{ }^{\mathrm{h}} \underline{\boldsymbol{m}}^{\frac{b}{2}} s_{1}
$$

where ${ }^{\mathrm{h}} \underline{\boldsymbol{\theta}}^{0}={ }^{\mathrm{h}} \underline{\boldsymbol{\theta}}\left(s_{1}=0\right)$, and periodicity for $\underline{\boldsymbol{\theta}}^{\text {per }}$ enforces:

$$
{ }^{\mathrm{h}} \boldsymbol{G} \cdot \Delta^{\mathrm{h}} \underline{\boldsymbol{\theta}}={ }^{\mathrm{h}} \underline{\boldsymbol{m}}^{\frac{b}{2}} b
$$

where $\Delta^{\mathrm{h}} \underline{\boldsymbol{\theta}}={ }^{\mathrm{h}} \underline{\boldsymbol{\theta}}(0)-{ }^{\mathrm{h}} \underline{\boldsymbol{\theta}}(b)$. Since $\underline{\boldsymbol{\theta}}^{\text {per }}$ is periodic, $\Delta^{\mathrm{h}} \underline{\boldsymbol{\theta}}$ is fully determined by $\underline{\boldsymbol{\Theta}}^{K L}$ defined in Equation 13 and we have:

$$
\Delta^{\mathrm{h}} \underline{\boldsymbol{\theta}}=\left(\begin{array}{c}
-b \chi_{12} \\
b \chi_{11} \\
0
\end{array}\right)
$$

Thus Equation B.6 yields:

$$
{ }^{\mathrm{h}} \underline{\boldsymbol{m}}^{\frac{b}{2}}=\left(\begin{array}{c}
-G \mathcal{J} \chi_{12} \\
E \mathcal{I}_{2} \chi_{11} \\
0
\end{array}\right) \mathrm{h}
$$

The constitutive equation for the translations leads to:

$$
{ }^{\mathrm{h}} \underline{\boldsymbol{u}}-{ }^{\mathrm{h}} \underline{\boldsymbol{u}}^{0}={ }^{\mathrm{h}} \underline{\sim}^{-1} \cdot{ }^{\mathrm{h}} \underline{\boldsymbol{r}}^{0} s_{1}-\underline{\boldsymbol{e}}_{1} \times\left({ }^{\mathrm{h}} \boldsymbol{G}^{-1} \cdot\left(-\underline{\boldsymbol{e}}_{1} \times{ }^{\mathrm{h}} \underline{\underline{r}}^{0}\left(\frac{s_{1}}{3}-\frac{b}{2}\right) \frac{s_{1}^{2}}{2}+{ }^{\mathrm{h}} \underline{\boldsymbol{m}}^{\frac{b}{2}} \frac{s_{1}^{2}}{2}\right)+{ }^{\mathrm{h}} \underline{\boldsymbol{\theta}}^{0} s_{1}\right)
$$

where ${ }^{\mathrm{h}} \underline{\boldsymbol{u}}^{0}={ }^{\mathrm{h}} \underline{\boldsymbol{u}}(0)$. The periodicity of ${ }^{\mathrm{h}} \underline{\boldsymbol{u}}^{\text {per }}$ enforces:

$$
\Delta^{\mathrm{h}} \underline{\boldsymbol{u}}+\frac{b}{2} \underline{\boldsymbol{e}}_{1} \times \Delta^{\mathrm{h}} \underline{\boldsymbol{\theta}}={ }^{\mathrm{h}} \underset{\sim}{\boldsymbol{k}} \cdot \underline{\boldsymbol{p}}^{\mathrm{h}}-b \underline{\boldsymbol{e}}_{1} \times{ }^{\mathrm{h}} \underline{\boldsymbol{\theta}}^{0}
$$

where

$$
{ }^{\mathrm{h}} \boldsymbol{\sim}=\left(\begin{array}{ccc}
\frac{b}{E \mathcal{S}} & 0 & 0 \\
0 & \frac{b}{G \mathcal{S}_{s, 2}}+\frac{b^{3}}{12 E \mathcal{I}_{3}} & 0 \\
0 & 0 & \frac{b}{G \mathcal{S}_{s, 3}}+\frac{b^{3}}{12 E \mathcal{I}_{2}}
\end{array}\right)
$$

and

$$
\Delta^{\mathrm{h}} \underline{\boldsymbol{u}}=\left(\begin{array}{c}
b e_{11} \\
b e_{12} \\
-b^{2} / 2 \chi_{11}
\end{array}\right)
$$


The first line of Equation B.9 leads directly to the traction in beam "h":

$$
{ }^{\mathrm{h}} r_{1}^{0}=E \mathcal{S} e_{11}
$$

Similar analysis for beam "v" with:

$$
\Delta^{\mathrm{v}} \underline{\boldsymbol{u}}=\left(\begin{array}{c}
b e_{22} \\
-b e_{12} \\
-b^{2} / 2 \chi_{22}
\end{array}\right)_{v} \text { and } \quad \Delta^{\mathrm{v}} \underline{\boldsymbol{\theta}}=\left(\begin{array}{c}
b \chi_{12} \\
b \chi_{22} \\
0
\end{array}\right)_{v}
$$

leads to:

$$
{ }^{\mathrm{v}} \underline{\boldsymbol{m}}^{\frac{b}{2}}=\left(\begin{array}{c}
G \mathcal{J} \chi_{12} \\
E \mathcal{I}_{2} \chi_{22} \\
0
\end{array}\right)_{\mathrm{v}}
$$

and

$$
{ }^{\mathrm{v}} r_{1}^{0}=E \mathcal{S} e_{22}
$$

Displacement continuity at node. There remain ${ }^{\mathrm{h}} r_{2}^{0}=-{ }^{\mathrm{v}} r_{2}^{0}$ components to derive. Rotation field continuity enforces: ${ }^{\mathrm{h}} \underline{\boldsymbol{\theta}}^{0}={ }^{\mathrm{v}} \underline{\boldsymbol{\theta}}^{0}$. Using this with Equation B.9 and its counterpart for beam "v" leads to:

$$
{ }^{\mathrm{h}} r_{2}^{0}=\frac{b e_{12}}{\frac{b}{G \mathcal{S}_{s, 2}}+\frac{b^{3}}{12 E \mathcal{I}_{3}}}
$$

Result. The preceding results are summarized in the following localization field:

$$
\begin{aligned}
& { }^{\mathrm{h}} \underline{\boldsymbol{r}}^{K L}=\left(\begin{array}{c}
E \mathcal{E} e_{11} \\
\frac{b e_{12}}{\bar{G} \mathcal{S}_{s, 2}+\frac{b^{3}}{12 E \mathcal{I}_{3}}} \\
0
\end{array}\right)_{\mathrm{h}} \text { and } \quad{ }^{\mathrm{h}} \underline{\boldsymbol{m}}^{K L}=\left(\begin{array}{c}
-G \mathcal{J} \chi_{12} \\
\frac{E \mathcal{I}_{2} \chi_{11}}{\frac{b e_{12}}{G \mathcal{S}_{s, 2}}+\frac{b^{3}}{12 E \mathcal{I}_{3}}}\left(s_{1}-\frac{b}{2}\right)
\end{array}\right)_{\mathrm{h}} \\
& { }^{\mathrm{v}} \underline{\underline{K}}^{K L}=\left(\begin{array}{c}
E \mathcal{S} e_{22} \\
-\frac{b e_{12}}{\frac{b}{G \mathcal{S}_{s, 2}}+\frac{b 3}{12 E \mathcal{I}_{3}}} \\
0
\end{array}\right)_{\mathrm{v}} \quad \text { and } \quad{ }^{\mathrm{v}} \underline{\boldsymbol{m}}^{K L}=\left(\begin{array}{c}
G \mathcal{J} \chi_{12} \\
E \mathcal{I}_{2} \chi_{22} \\
-\frac{b e_{12}}{\frac{b}{G \mathcal{S}_{s, 2}}+\frac{b^{3}}{12 E \mathcal{I}_{3}}}\left(s_{1}-\frac{b}{2}\right)
\end{array}\right)
\end{aligned}
$$

The Kirchhoff-Love stiffness tensors are derived using Equation 17 which leads to the result in Equation (28). Finally, using the constitutive equations: $\underset{\sim}{\boldsymbol{N}}=\underset{\sim}{\boldsymbol{A}}: \underset{\sim}{\boldsymbol{e}}$ and $\underset{\sim}{M}=\underset{\sim}{\boldsymbol{D}}: \underset{\sim}{\chi}$ leads to the field localization given in Equation (27).

\section{Appendix C. Exact solution for the cylindrical bending of a $45^{\circ}$ lattice}

Effect of symmetries. The lattice configuration is symmetric with respect to $\left(A^{2 n}, \underline{e}_{2}, \underline{e}_{3}\right)$ plane and any $\left(A^{j}, \underline{e}_{1}, \underline{e}_{3}\right)$ plane. Thus, the results are derived for half the lattice (see Figure 6 ) and we have the following relation between fields in each set:

$$
\begin{aligned}
{ }^{+} \underline{\boldsymbol{r}} & ={\underset{\sim}{\boldsymbol{P}}}^{\mathcal{M}} \cdot{ }^{-} \underline{\boldsymbol{r}} \text { and }{ }^{+} \underline{\boldsymbol{m}}=-\underset{\sim}{\boldsymbol{P}}{ }^{\mathcal{M}} \cdot{ }^{-} \underline{\boldsymbol{m}} \\
{ }^{+} \underline{\boldsymbol{u}} & ={\underset{\sim}{\boldsymbol{P}}}^{\mathcal{M}} \cdot{ }^{-} \underline{\boldsymbol{u}} \text { and }{ }^{+} \underline{\boldsymbol{\theta}}=-\underset{\sim}{\boldsymbol{P}}{ }^{\mathcal{M}} \cdot{ }^{-} \underline{\boldsymbol{\theta}}
\end{aligned}
$$

where + denotes the stress in the first beam set and- denotes the stress in the second beam set. ${\underset{\sim}{\mathcal{M}}}^{\mathcal{M}}=$ $\left(\begin{array}{ccc}1 & 0 & 0 \\ 0 & -1 & 0 \\ 0 & 0 & 1\end{array}\right)$ is the symmetry matrix expressed in the main reference frame ( $\boldsymbol{m}$ and $\underline{\boldsymbol{\theta}}$ are pseudo vectors, which explains the sign).

Moreover the symmetry vanishes some displacement at nodes $A^{j}$. We have in the main reference frame $u_{2}^{j}=\theta_{1}^{j}=\theta_{3}^{j}=0$ where $u_{2}^{j}$ means $u_{2}\left(x^{j}\right)$ 


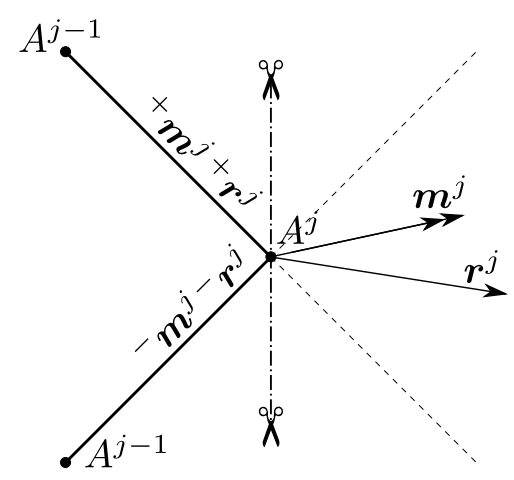

Figure C.12: Resultants and cutting plane at node $A^{j}$

Equilibrium at nodes. Cutting the lattice at node $A^{j}$ with $\left(A^{j}, \underline{\boldsymbol{e}}_{2}, \underline{e}_{3}\right)$ plane leads to the following equilibrium equations at nodes (see Figure C.12):

$$
\begin{gathered}
\underline{\boldsymbol{r}}^{j}={ }^{+} \underline{\boldsymbol{r}}^{j}+{ }^{-} \underline{\boldsymbol{r}}^{j} \\
\underline{\boldsymbol{m}}^{j}={ }^{+} \underline{\boldsymbol{m}}^{j}+{ }^{-} \underline{\boldsymbol{m}}^{j}
\end{gathered}
$$

where ${ }^{+} \underline{\boldsymbol{r}}^{j}={ }^{+} \underline{\boldsymbol{r}}\left(x^{j}\right)$ is the resultant on beam "+" at $x^{j} . \underline{\boldsymbol{r}}^{j}$ is the resultant through cutting plane.

Taking into account symmetry, this leads to:

$$
\begin{aligned}
& r_{1}^{j}=\sqrt{2}\left({ }^{+} r_{1}^{j}-{ }^{+} r_{2}^{j}\right), \quad r_{2}^{j}=0, \quad r_{3}^{j}=2{ }^{+} r_{3}^{j} \\
& m_{1}^{j}=0, \quad m_{2}^{j}=\sqrt{2}\left({ }^{+} m_{2}^{j}+{ }^{+} m_{1}^{j}\right), \quad m_{3}^{j}=0
\end{aligned}
$$

where ${ }^{+} r_{i}^{j}$ and ${ }^{-} r_{i}^{j}$ are the components of ${ }^{+} \underline{\boldsymbol{r}}^{j}$ and ${ }^{-} \underline{\boldsymbol{r}}^{j}$ taken in the local reference frame of the beam and $r_{i}^{j}$ are the components of $\boldsymbol{r}^{j}$ taken in the main reference frame.

Macroscopic equilibrium. Using global equilibrium at node $A^{j}$, it is possible to derive resultants and moments $\underline{\boldsymbol{r}}^{j}$ and $\underline{\boldsymbol{m}}^{j}$ through the cutting plane. We have:

$$
r_{1}^{j}=0, \quad r_{3}^{j}=-2 \sqrt{2} \frac{k_{0}}{\kappa} \cos _{j}, \quad m_{2}^{j}=-2 \sqrt{2} \frac{k_{0}}{\kappa^{2}} \sin _{j}
$$

in the main reference frame, where $\cos _{j}=\cos \kappa x^{j}$.

Individual beam problem to solve. Combining preceding equations, it is possible to derive boundary condition to apply to each beam of set "+" in the local reference frame:

$$
\begin{gathered}
{ }^{+} r_{1}^{j}=-{ }^{+} r_{2}^{j}, \quad{ }^{+} r_{3}^{j}=-\sqrt{2} \frac{k_{0}}{\kappa} \cos { }_{j}, \quad{ }^{+} m_{2}^{j}+{ }^{+} m_{1}^{j}=-2 \frac{k_{0}}{\kappa^{2}} \sin _{j}, \quad{ }^{+} m_{3}^{j}=0 \\
{ }^{+} u_{1}^{j}=-{ }^{+} u_{2}^{j}, \quad{ }^{+} \theta_{1}^{j}={ }^{+} \theta_{2}^{j}, \quad{ }^{+} \theta_{3}^{j}=0
\end{gathered}
$$

To this must be added $+u_{3}^{0}=0$ for the simple support and ${ }^{+} \theta_{2}^{2 n}=0$ for symmetry condition at mid span.

With these boundary conditions and the beam model introduced in Section 2.2.1 one gets the result given in Section 4.2.1

\section{Appendix D. Homogenization solutions for cylindrical bending}

Appendix D.1. Lattice at $0^{\circ}$

The cylindrical bending of a Bending-Gradient plate loaded with $\underline{\boldsymbol{p}}=p_{0} \sin \kappa x_{1} \underline{\boldsymbol{e}}_{3}$ is solved in details in [13]. 
Macroscopic deflection. The macroscopic deflection from [13] is:

$$
U_{3}^{B G}=-\frac{p_{0}}{\kappa^{4}}\left(g_{11}-{ }^{T} \underline{\underline{g}} \cdot \underline{\sim}^{-1} \cdot \underline{g}\right) \sin \kappa x_{1}
$$

where

$$
\underset{\sim}{\hat{g}}=[\underset{\sim}{\boldsymbol{d}}]+\kappa^{2} \underset{\sim}{\hat{\boldsymbol{f}}}, \quad \underset{\sim}{\boldsymbol{g}}=\left(\begin{array}{ll}
g_{22} & g_{23} \\
g_{23} & g_{33}
\end{array}\right), \quad \underline{\boldsymbol{g}}=\left(\begin{array}{c}
g_{12} \\
g_{13}
\end{array}\right) .
$$

The matrix $[\underset{\sim}{\boldsymbol{d}}]$ is Kirchhoff-Love flexural stiffness and the $3 \times 3$ matrix $\underset{\sim}{\hat{f}}$ is the upper-left $3 \times 3$-block of $[\underline{f}]$.

For the configuration at $0^{\circ},[\underset{\sim}{\boldsymbol{d}}]$ is given by Equation 28 and $\underset{\sim}{\boldsymbol{f}}$ by Equation $[36$, thus:

$$
\hat{\sim}=\frac{b}{G \mathcal{S}_{s, 3}}\left(\begin{array}{ccc}
1 & 0 & 0 \\
0 & 0 & 0 \\
0 & 0 & \frac{1+\eta}{2}
\end{array}\right)
$$

With these values for $[\underset{\sim}{\boldsymbol{d}}]$ and $\underset{\sim}{\hat{f}}$, we have:

$$
U_{3}^{B G}=-\frac{k_{0}}{\kappa^{4}}\left(\frac{1}{E \mathcal{I}_{2}}+\frac{\pi^{2}}{L^{2} G \mathcal{S}_{s, 3}}\right) \sin \kappa x_{1}
$$

Macroscopic stress. Furthermore, the solution for the bending moment and the bending gradient from [13] are:

$$
\begin{aligned}
& {[\underset{\sim}{\boldsymbol{M}}]=\left(\begin{array}{c}
M_{11} \\
M_{22} \\
\sqrt{2} M_{12}
\end{array}\right)=\left(\begin{array}{c}
-1 \\
\underline{\boldsymbol{g}}^{-1} \cdot \underline{\boldsymbol{g}}
\end{array}\right) \frac{p_{0}}{\kappa^{2}} \sin \kappa x_{1}} \\
& {[\underline{\boldsymbol{R}}]=\left(\begin{array}{c}
R_{111} \\
R_{221} \\
\sqrt{2} R_{121} \\
R_{112} \\
R_{222} \\
\sqrt{2} R_{122}
\end{array}\right)=\left(\begin{array}{c}
-1 \\
{\underset{\sim}{\boldsymbol{g}}}^{-1} \cdot \underline{g} \\
0 \\
0 \\
0
\end{array}\right) \frac{p_{0}}{\kappa} \cos \kappa x_{1}}
\end{aligned}
$$

It becomes:

$$
[\underset{\sim}{\boldsymbol{M}}]=\left(\begin{array}{c}
-1 \\
0 \\
0
\end{array}\right) \frac{k_{0}}{b \kappa^{2}} \sin \kappa x_{1} \quad \text { and } \quad[\underline{\boldsymbol{R}}]=\left(\begin{array}{c}
-1 \\
0 \\
0 \\
0 \\
0 \\
0
\end{array}\right) \frac{k_{0}}{b \kappa} \cos \kappa x_{1}
$$

Localization. We introduce these values in the localization Equations 27 and 34 and get the following local generalized stress fields:

$$
\begin{aligned}
& { }^{\mathrm{h}} m_{2}^{B G}=-\frac{k_{0}}{\kappa^{2}} \sin \kappa x_{1} \\
& { }^{\mathrm{h}} r_{3}^{B G}=-\frac{k_{0}}{\kappa} \cos \kappa x_{1}
\end{aligned}
$$

Reissner-Mindlin approximation. The Reissner-Mindlin approximation is easily derived while repeating the previous steps and setting $\boldsymbol{f}=\frac{b}{G \mathcal{S}_{s, 3}} \underset{\sim}{\boldsymbol{i}} \cdot \underset{\sim}{\boldsymbol{i}}$. This leads to exactly the same result as the Bending-Gradient approximation. 
Kirchhoff-Love approximation. The Kirchhoff-Love approximation is derived from the Bending-Gradient approximation while setting $\boldsymbol{f}=0$. The approximate deflection becomes:

$$
U_{3}^{K L}=-\frac{k_{0}}{\kappa^{4} E \mathcal{I}_{2}} \sin \kappa x_{1}
$$

Furthermore, there is no localization related to shear forces and then:

$$
\begin{gathered}
{ }^{\mathrm{h}} r_{3}^{K L}=0 \\
{ }^{\mathrm{h}} m_{2}^{K L}=-\frac{k_{0}}{\kappa^{2}} \sin \kappa x_{1}
\end{gathered}
$$

Appendix D.2. Lattice at $45^{\circ}$

Appendix D.2.1. Bending-Gradient approximation

The approximation for the lattice at $45^{\circ}$ is derived following a similar procedure as in the previous section. However one have to take into account the rotation of the lattice. This affects both the constitutive equation of the Bending-Gradient model but also the localization in the unit-cell. Full details about rotation of $\boldsymbol{R}$ and its constitutive equation are provided in $[13$.

Macroscopic fields. The flexural stiffness rotated by $45^{\circ}$ is now:

$$
[\underset{\approx}{\boldsymbol{d}}]=\frac{b}{2 E \mathcal{I}_{2}} \frac{1+\alpha}{\alpha}\left(\begin{array}{ccc}
1 & -\frac{1-\alpha}{1+\alpha} & 0 \\
-\frac{1-\alpha}{1+\alpha} & 1 & 0 \\
0 & 0 & \frac{2 \alpha}{1+\alpha}
\end{array}\right)
$$

where $\alpha=\frac{G \mathcal{J}}{E \mathcal{I}_{2}}$. The matrix $\underset{\sim}{\hat{f}}$ becomes also:

$$
\hat{\sim} \underset{\sim}{\boldsymbol{f}}=\frac{b}{G \mathcal{S}_{s, 3}}\left(\begin{array}{ccc}
1+\frac{\eta}{4} & -\frac{\eta}{4} & 0 \\
-\frac{\eta}{4} & \frac{\eta}{4} & 0 \\
0 & 0 & 1 / 2
\end{array}\right)
$$

Inserting this in Equation D.1 leads to the following macroscopic deflection:

$$
U_{3}=-U^{K L}\left(1+\frac{\gamma^{2}}{\alpha}\left(\frac{(1+\alpha)^{2}}{\eta}+\frac{\alpha^{2}}{1+\gamma^{2}}\right)\right) \sin \kappa x_{1}
$$

where $U^{K L}=\frac{4 k_{0}}{\kappa^{4} E \mathcal{I}_{2}(1+\alpha)}$ is the Kirchhoff-Love maximum deflection and $\gamma^{2}=\frac{\kappa^{2} b^{2}}{24}$ is the ratio between the size of the unit-cell and the span.

The bending moment writes as:

$$
[\boldsymbol{M}]=-\left(\begin{array}{c}
1 \\
\frac{1}{1+\gamma^{2}}\left(\frac{1-\alpha}{1+\alpha}+\gamma^{2}\right) \\
0
\end{array}\right) \frac{2 k_{0}}{b \kappa^{2}} \sin \kappa x_{1}
$$

and the bending gradient:

$$
[\underline{\boldsymbol{R}}]=-\left(\begin{array}{c}
1 \\
\frac{1}{1+\gamma^{2}}\left(\frac{1-\alpha}{1+\alpha}+\gamma^{2}\right) \\
0 \\
0 \\
0 \\
0
\end{array}\right)_{h}
$$


Localization of the solution. In order to relocalize macroscopic fields, we rotate them in the reference frame of beams "+" (rotation of $+45^{\circ}$, cf. [13]). We get:

$$
\begin{gathered}
{[\underset{\sim}{M}]=-\frac{1}{1+\gamma^{2}}\left(\begin{array}{c}
\frac{1}{1+\alpha}+\gamma^{2} \\
\frac{1}{1+\alpha}+\gamma^{2} \\
-\frac{\sqrt{2} \alpha}{1+\alpha}
\end{array}\right)_{+} \frac{2 k_{0}}{b \kappa^{2}} \sin \kappa x_{1}} \\
{[\underline{\boldsymbol{R}}]=-\frac{1}{\sqrt{2}\left(1+\gamma^{2}\right)}\left(\begin{array}{c}
\frac{1}{1+\alpha}+\gamma^{2} \\
\frac{1}{1+\alpha}+\gamma^{2} \\
-\frac{\sqrt{2} \alpha}{1+\alpha} \\
\frac{1}{1+\alpha}+\gamma^{2} \\
\frac{1}{1+\alpha}+\gamma^{2} \\
\frac{\sqrt{2} \alpha}{1+\alpha}
\end{array}\right)_{+} \frac{2 k_{0}}{b \kappa} a \cos \kappa x_{1}}
\end{gathered}
$$

We introduce these values in the localization Equations 27 and 34 and get the local generalized stress fields for beams "+" given in Equations [54 and 55.

\section{Appendix D.2.2. Reissner-Mindlin approximation}

Macroscopic fields. Again, the Reissner-Mindlin approximation is derived taking $\underset{\sim}{\boldsymbol{f}}=\frac{b}{G \mathcal{S}_{s, 3}} \underset{\sim}{\boldsymbol{i}} \cdot \underset{\sim}{\boldsymbol{i}}$ in Equation D.14 and following previous steps. We have the macroscopic deflection:

$$
U_{3}^{R M}=-U^{K L}\left(1+\gamma^{2} \frac{(1+\alpha)^{2}}{\eta \alpha}\right) \sin \kappa x_{1}
$$

The macroscopic bending moment is:

$$
[\underset{\sim}{M}]=-\left(\begin{array}{c}
1 \\
\frac{1-\alpha}{1+\alpha} \\
0
\end{array}\right) \frac{2 k_{0}}{b \kappa^{2}} \sin \kappa x_{1}
$$

Shear forces are derived taking the divergence of this:

$$
\underline{Q}=-\frac{2 k_{0}}{\kappa} \cos \kappa x_{1} \underline{e}_{1}
$$

Localization. In order to relocalize macroscopic fields, we rotate them in the reference frame of beams "+" $\left(\right.$ rotation of $\left.+45^{\circ}\right)$.

$$
\begin{aligned}
& {[\underset{\sim}{M}]=-\left(\begin{array}{c}
\frac{1}{1+\alpha} \\
\frac{1}{1+\alpha} \\
-\frac{\sqrt{2} \alpha}{1+\alpha}
\end{array}\right)_{+} \frac{2 k_{0}}{b \kappa^{2}} \sin \kappa x_{1}} \\
& \underline{Q}=-\frac{2 k_{0}}{\kappa} \cos \kappa x_{1}\left(\frac{{ }^{+} \underline{\boldsymbol{t}}}{\sqrt{2}}-\frac{{ }^{+} \underline{\boldsymbol{n}}}{\sqrt{2}}\right)
\end{aligned}
$$

Inserting this in the Reissner-Mindlin localization field 35 leads to:

$$
{ }^{+} \underline{\underline{\boldsymbol{r}}}^{R M}=-\frac{\sqrt{2} k_{0}}{\kappa} \cos \kappa x_{1} \underline{\boldsymbol{e}}_{3} \quad \text { and } \quad{ }^{+} \underline{\boldsymbol{m}}^{R M}=-\frac{m^{*}}{1+\alpha}\left(\begin{array}{c}
\alpha \sin \kappa x_{1} \\
\sin \kappa x_{1} \\
0
\end{array}\right)_{+}
$$




\section{Appendix D.2.3. Kirchhoff-Love approximation}

Kirchhoff-Love approximation is derived setting $\boldsymbol{f}=0$ in the Bending-Gradient approach. This leads to:

$$
U_{3}^{K L}=-U^{K L} \sin \kappa x_{1}
$$

Localized fields are limited to the Kirchhoff-Love contribution:

$$
{ }^{+} \underline{\boldsymbol{r}}^{K L}=\underline{\mathbf{0}} \text { and } \quad{ }^{+} \underline{\boldsymbol{m}}^{K L}=-\frac{m^{*}}{1+\alpha}\left(\begin{array}{c}
\alpha \sin \kappa x_{1} \\
\sin \kappa x_{1} \\
0
\end{array}\right)_{+}
$$

[1] P. G. Ciarlet, P. Destuynder, Justification Of The 2-Dimensional Linear Plate Model, Journal de Mecanique 18 (1979) 315-344.

[2] D. Caillerie, Thin elastic and periodic plates, Mathematical Methods in the Applied Sciences 6 (1984) 159-191.

[3] E. Reissner, The effect of transverse shear deformation on the bending of elastic plates, Journal of Applied Mechanics 12 (1945) 69-77.

[4] J. M. Whitney, Stress Analysis of Thick Laminated Composite and Sandwich Plates, Journal of Composite Materials 6 (1972) 426-440.

[5] J. N. Reddy, On refined computational models of composite laminates, International Journal for Numerical Methods in Engineering 27 (1989) 361-382.

[6] A. K. Noor, M. Malik, An assessment of five modeling approaches for thermo-mechanical stress analysis of laminated composite panels, Computational Mechanics 25 (2000) 43-58.

[7] E. Carrera, Theories and finite elements for multilayered, anisotropic, composite plates and shells, Archives of Computational Methods in Engineering 9 (2002) 87-140.

[8] T.-K. Nguyen, K. Sab, G. Bonnet, Shear Correction Factors for Functionally Graded Plates, Mechanics of Advanced Materials and Structures 14 (2007) 567-575.

[9] T.-K. Nguyen, K. Sab, G. Bonnet, First-order shear deformation plate models for functionally graded materials, Composite Structures 83 (2008) 25-36.

[10] T. Lewiski, Effective models of composite periodic platesI. Asymptotic solution, International Journal of Solids and Structures 27 (1991) 1155-1172.

11] A. Cecchi, K. Sab, A homogenized ReissnerMindlin model for orthotropic periodic plates: Application to brickwork panels, International Journal of Solids and Structures 44 (2007) 6055-6079.

[12] A. Lebée, K. Sab, A Bending-Gradient model for thick plates. Part I: Theory, International Journal of Solids and Structures 48 (2011) 2878-2888.

[13] A. Lebée, K. Sab, A Bending-Gradient model for thick plates, Part II: Closed-form solutions for cylindrical bending of laminates, International Journal of Solids and Structures 48 (2011) 2878-2888.

[14] A. Lebée, K. Sab, Homogenization of thick periodic plates: Application of the Bending-Gradient plate theory to a folded core sandwich panel, International Journal of Solids and Structures 49 (2012) 2778-2792.

[15] A. Lebée, K. Sab, Homogenization of cellular sandwich panels, Comptes Rendus Mécanique 340 (2012) $320-337$.

[16] V. S. Deshpande, N. A. Fleck, Collapse of truss core sandwich beams in 3-point bending, International Journal of Solids and Structures 38 (2001) 6275-6305.

[17] V. S. Deshpande, N. A. Fleck, M. Ashby, Effective properties of the octet-truss lattice material, Journal of the Mechanics and Physics of Solids 49 (2001) 1747-1769.

[18] J. C. Wallach, L. J. Gibson, Mechanical behavior of a three-dimensional truss material, International Journal of Solids and Structures 38 (2001) 7181-7196.

[19] N. Wicks, J. W. Hutchinson, Optimal truss plates, International Journal of Solids and Structures 38 (2001) $5165-5183$.

[20] C. Douthe, O. Baverel, Design of nexorades or reciprocal frame systems with the dynamic relaxation method, Computers \& Structures 87 (2009) 1296-1307.

[21] C. Douthe, O. Baverel, J.-F. Caron, Gridshell in composite materials: Towards wide span shelters, Journal of the International Association for Shell and Spatial Structures 48 (2007) 175-180.

[22] F. Pradel, K. Sab, Homogenization of discrete media, Journal de Physique. IV : JP 8 (1998) 317-324.

[23] C. Florence, K. Sab, A rigorous homogenization method for the determination of the overall ultimate strength of periodic discrete media and an application to general hexagonal lattices of beams, European Journal of Mechanics - A/Solids 25 (2006) 72-97.

[24] C. Boutin, Microstructural effects in elastic composites, International Journal of Solids and Structures 33 (1996) 10231051. 